TRANSACTIONS OF THE

AMERICAN MATHEMATICAL SOCIETY

Volume 360, Number 9, September 2008, Pages 4889-4912

S 0002-9947(08)04403-6

Article electronically published on April 9, 2008

\title{
COMPARISON THEOREMS FOR O-MINIMAL SINGULAR (CO)HOMOLOGY
}

\author{
MÁRIO J. EDMUNDO AND ARTHUR WOERHEIDE
}

\begin{abstract}
Here we show the existence of the o-minimal simplicial and singular (co)homology in o-minimal expansions of real closed fields and prove several comparison theorems for o-minimal (co)homology theories.
\end{abstract}

\section{INTRODUCTION}

As it is known o-minimal geometry is a wide ranging generalization of semialgebraic, sub-analytic and sub-pfaffian geometry. In this paper we will work over an o-minimal expansion $\mathcal{N}$ of an ordered ring (necessarily a real closed field) and definable means definable in $\mathcal{N}$ possibly with parameters.

Associated to the first-order theory $\operatorname{Th}(\mathcal{N})$ of $\mathcal{N}$ over $N$ we have the categories of definable sets and of definably compact definable sets (or equivalently of closed and bounded definable sets; see $[\mathrm{ps}]$ ). The morphisms on these categories are continuous definable maps. For basic o-minimality and the notion of definable sets and definable maps we refer the reader to vdd.

By [KPS] and the first-order logic compactness theorem, for each infinite cardinal $\kappa$, the theory $\operatorname{Th}(\mathcal{N})$ has a model of cardinality $\kappa$ and any such model is also an o-minimal structure. Moreover, by $[\mathrm{Sh}]$, for every $\kappa>\max \left\{\aleph_{0},|\operatorname{Th}(\mathcal{N})|\right\}$, there are up to isomorphism $2^{\kappa}$ models $\mathcal{M}$ of $\operatorname{Th}(\mathcal{N})$ such that $|M|=\kappa$. A model $\mathcal{M}$ of $\operatorname{Th}(\mathcal{N})$ determines the categories of definable sets and of definably compact definable sets defined over $M$. Since we are assuming that $\operatorname{Th}(\mathcal{N})$ is the theory of $\mathcal{N}$ over $N$, the model $\mathcal{M}$ detemines a functor from the category of definable sets (resp., of definably compact definable sets) (defined over $N$ ) into the category of definable sets (resp., of definably compact definable sets) defined over $M$. In o-minimal geometry we are interested in developing a geometry which is invariant under this functor.

For example, if $\mathcal{N}$ is the field $\mathbb{R}$ of real numbers (resp., the expansion $\mathbb{R}_{\mathrm{an}}$ of $\mathbb{R}$ by all globally analytic functions), then the geometry of the category of definable sets is semi-algebraic geometry (resp., global sub-analytic geometry). While the geometry on models of $\mathbb{R}$, which are exactly the real closed fields, is already well developed within semi-algebraic geometry (see [BCR]), the geometry in arbitrary models of $\mathbb{R}_{\mathrm{an}}$ and of other o-minimal structures on the real numbers such

Received by the editors November 13, 2003 and, in revised form, December 16, 2004, November 3, 2005, July 17, 2006, and September 8, 2006.

2000 Mathematics Subject Classification. Primary 03C64, 55N10.

Key words and phrases. O-minimal singular homology and cohomology.

This research was supported by the FCT grant SFRH/BPD/6015/2001 and partially by the European Research and Training Network HPRN-CT-2001-00271 RAAG. 
as $\mathbb{R}_{\text {exp }}, \mathbb{R}_{\text {an, exp }}, \mathbb{R}_{\text {an* }}, \mathbb{R}_{\text {an*,exp }}$, etc. is still being developed, and the present paper is a contribution in this direction. For the o-minimality of the structures $\mathbb{R}_{\mathrm{an}}$, $\mathbb{R}_{\text {exp }}, \mathbb{R}_{\text {an, exp }}, \mathbb{R}_{a n^{*}}, \mathbb{R}_{\text {an*, exp }}$ see resp., dd], [w], dm, ds1] and ds2.

We should also point out that we will be working in a larger generality since we will not assume that the first order theory $\operatorname{Th}(\mathcal{N})$ of $\mathcal{N}$ has a model with the order type of the reals.

An important tool for developing the geometry of definable sets would be of course a homology and cohomology theory for this category. Such (co)homology theories are defined by the Eilenberg-Steenrod (co)homology axioms adapted to the o-minimal setting (see [Wo or Sections 3 and 6 here).

In the thesis [Wo, the second author constructs the o-minimal simplicial homology for the category of definably compact sets and the o-minimal singular homology for the category of definable sets. From this one easily constructs the o-minimal simplicial cohomology and the o-minimal singular cohomology (see [e] or Section 6 here). Note that since the proof of the existence of the classical topological simplicial (resp., singular) homology theory uses local compactness of closed simplicial complexes, namely for proving the simplicial approximation theorem (resp., the local compactness of the standard simplices, namely in the proof of the excision axiom), the classical techniques do not generalize to the o-minimal case.

Since the thesis Wo has not been published, the construction of the o-minimal singular and simplicial homology theory will be included here in Sections 4 and 5 .

As observed in $\mathrm{Wo}$, the definable triangulation theorem ( $\mathrm{vdd}]$ ) and a purely algebraic argument imply as in the classical topological case in dp, Chapter 6 (6.3), the following (where here and below, $Q$ is a module over a commutative ring $R$ with a unit element):

Theorem 1.1. Any two o-minimal (co)homology theories in the category of definably compact definable sets with coefficients in $Q$ are isomorphic. In particular, any o-minimal (co)homology theory in the category of definably compact definable sets with coefficients in $Q$ is isomorphic to the o-minimal simplicial (co)homology with coefficients in $Q$.

Note that Theorem 1.1 is about definably compact definable sets which are analogues of the semi-algebraically complete sets from real algebraic geometry and are not in general compact sets. Thus this is not a consequence of the classical uniqueness theorem for homology theories on the category of compact topological triangulable spaces given in [es], Chapter III, Theorem 10.1.

One of the main results of the paper is the following generalization of Theorem 1.1 to the category of definable sets. Although definable sets are also definably triangulable ( $\mathrm{vdd}$ ) this result is not the same as the classical result [es], Chapter III, Theorem 10.1, since there simplicial complexes are compact, unlike in the ominimal case where they are not assumed to be closed.

Theorem 1.2. Any two o-minimal (co)homology theories on the category of definable sets with coefficients in $Q$ are isomorphic. In particular, any o-minimal (co)homology theory in the category of definable sets with coefficients in $Q$ is isomorphic to the o-minimal singular (co)homology with coefficients in $Q$.

This uniqueness theorem implies the natural invariance of the o-minimal singular (co)homology in elementary extensions and in o-minimal expansions of $\mathcal{N}$. Also, in the case that $\mathcal{N}$ is an o-minimal expansion of the field of real numbers, 
it follows that the o-minimal singular (co)homology is naturally isomorphic to the usual topological singular (co)homology. Here is the homology version of what we mean:

Corollary 1.3. Suppose that $\mathcal{M}$ is an elementary extension or an o-minimal expansion of $\mathcal{N}$. Let $A \subseteq X$ and $B \subseteq Y$ be definable sets over $N$ and $f: X \longrightarrow Y a$ continuous definable map over $N$ such that $f(A) \subseteq B$. Then we have isomorphisms $i_{*}^{\mathcal{M}}: H_{*}(X, A ; Q) \longrightarrow H_{*}(X(M), A(M) ; Q)$ making the diagram

$$
\begin{aligned}
H_{*}(X, A ; Q) & \stackrel{f_{*}}{\longrightarrow} & H_{*}(Y, B ; Q) \\
\downarrow^{i_{*}^{\mathcal{M}}} & & \downarrow^{i_{*}^{\mathcal{M}}} \\
H_{*}(X(M), A(M) ; Q) & \stackrel{\left(f^{M}\right)_{*}}{\longrightarrow} & H_{*}(Y(M), B(M) ; Q)
\end{aligned}
$$

commutative.

It is possible to obtain a variant of Theorem 1.2 that does not assume ominimality. This is a generalization of the classical result [es], Chapter III, Theorem 10.1, since there simplicial complexes are compact, unlike here where they are not assumed to be closed.

Theorem 1.4. Any two (co)homology theories on the category of finitely triangulable topological spaces over the real numbers with coefficients in $Q$ are isomorphic.

The natural invariance of the o-minimal simplicial (co)homology in elementary extensions was already pointed out in [bo] and [e] as it follows from the construction of the o-minimal simplicial (co)homology.

The existence of a semi-algebraic (co)homology theory is proved in [D1] (see also [D2 and $\mathrm{dk}$ ) in a rather complicated way, via sheaf cohomology theory with constant coefficients for the semi-algebraic site. Using the semi-algebraic triangulation theorem from $\mathrm{dk}$, it is proved in [D1] (see also $[\mathrm{dk}$ ) that the semi-algebraic (co)homology groups of definably compact definable sets coincide with the simplicial (co)homology groups of the corresponding simplicial complexes. Furthermore, in [D2, the natural invariance of semi-algebraic (co)homology theory in elementary extensions is proved.

In $[\mathrm{k}$, Knebusch states that it would be desirable to have a more elementary proof of the existence of the semi-algebraic singular (co)homology. The thesis Wo provides such elementary proofs.

The main theorems of the paper are a combination of homological algebra, ominimality and pure model theory. From homological algebra we need, among other things, the method of acyclic models and the theorem on removing cells. From ominimality the main result we need is the definable triangulation theorem, and from model theory we require the reader's familiarity with the notions of models and elementary extensions of models of a theory.

\section{TOOLS FROM HOMOLOGICAL ALGEBRA}

In this section we recall basic notions from homological algebra together with the method of acyclic models and the theorem on removing cells. Here we will follow [d, Chapter II and Chapter VI, Section 11, where the reader can find all the details.

Below $R$ will be a commutative ring with unit, Comp denotes the category of chain complexes of $R$-modules and $\widetilde{\mathbf{C o m p}}$ the category of augmented chain 
complexes of $R$-modules, i.e., the subcategory whose objects are all those chain complexes $E_{*}=\left(E_{*}, \partial_{*}\right)$ with $E_{-1}=\mathbb{Z}$, and $E_{n}=0$ for $n<-1$, and whose morphisms are those chain maps $f$ between such chain complexes with $f_{-1}=1_{\mathbb{Z}}$.

We say that a chain complex $E_{*}=\left(E_{*}, \partial_{*}\right)$ is acyclic if $H_{n}\left(E_{*}\right)=\operatorname{Ker} \partial_{n} / \operatorname{Im} \partial_{n+1}$ $=0$ for all $n \in \mathbb{Z}$. The chain complex $E_{*}$ is said to be free if $E_{n}$ is a free $R$-module for all $n \in \mathbb{Z}$. A family $U_{*}=\left(U_{n}\right)_{n \in \mathbb{Z}}$ is a basis of $E_{*}$ if $U_{n}$ is a basis for $E_{n}$ for each $n \in \mathbb{Z}$; if $U_{*}$ is a basis for $E_{*}, s \in U_{n}$ and $t \in E_{n}$, we say that $s$ is involved in $t$ if, when $t$ is written as an $R$-linear combination of elements of $U_{n}, s$ has a nonzero coefficient.

A subcomplex $F_{*}=\left(F_{*}, \partial_{*}\right)$ is an adequate subcomplex of $E_{*}$ if (i) whenever $z \in \operatorname{Ker} \partial_{n}$, then there is $z^{\prime} \in \operatorname{Ker} \partial_{n} \cap F_{n}$ such that $z-z^{\prime} \in \operatorname{Im} \partial_{n+1}$ and (ii) if $z^{\prime} \in \operatorname{Ker} \partial_{n} \cap F_{n}$ and $z^{\prime}=\partial_{n+1} c$ for some $c \in E_{n+1}$, then there is $c^{\prime} \in F_{n+1}$ with $z^{\prime}=\partial_{n+1} c^{\prime}$.

Note that if $F_{*}$ is an adequate subcomplex of $E_{*}$, then the inclusion $i: F_{*} \longrightarrow E_{*}$ induces isomorphisms $H_{n}(i): H_{n}\left(F_{*}\right) \longrightarrow H_{n}\left(E_{*}\right)$ for all $n \in \mathbb{Z}$. Later we will need the following result from [Wo].

Theorem 2.1 (Removing cells). Let $E_{*}$ be a chain complex with basis $U_{*}, s \in U_{n}$ and $t \in U_{n+1}$ such that $s=\partial_{n+1} t+r$ with $s$ not involved in $r$ and such that $s$ is not involved in $\partial_{n+1} t^{\prime}$ for all $t^{\prime} \in U_{n+1}-\{t\}$. Let $F_{m}=E_{m}$ for $m \neq n, n+1$, $F_{n}=\left\langle U_{n}-\{s\}\right\rangle$ and $F_{n+1}=\left\langle U_{n+1}-\{t\}\right\rangle$. Then $F_{*}$ with the restricted boundary maps forms an adequate subcomplex of $E_{*}$.

Proof. It is easy to see that $F_{*}$ is indeed a subcomplex of $E_{*}$, i.e., $\partial_{*}\left(F_{m}\right) \subseteq F_{m-1}$ for all $m \in \mathbb{Z}$. The case $m=n+2$ is the only case that needs to be checked. But if $r$ is a $(n+2)$-cell such that $t$ is involved in $\partial_{n+2} r$, then $s$ is involved in $\partial_{n+1} \circ \partial_{n+2} r$, so that $\partial_{n+1} \circ \partial_{n+2} r \neq 0$, which is a contradiction.

It remains to verify conditions (i) and (ii) of the definition of an adequate subcomplex. This is trivial for all dimensions other than $n+1, n$ and $n-1$. In dimension $n+1$, condition (i) follows from the fact that if $z \in \operatorname{Ker} \partial_{n}$, then $z$ does not involve $t$ and so $z \in \operatorname{Ker}_{n} \cap F_{n}$; on the other hand, condition (ii) follows from the fact that $F_{n+2}=E_{n+2}$.

We now consider the case of dimension $n$. We start with condition (i). Let $z \in \operatorname{Ker} \partial_{n}$. Write $z=m s+\beta$, with $s$ not involved in $\beta$. Let $z^{\prime}=z-m \partial_{n+1} t=$ $\beta+m r$. Then $\partial_{n} z^{\prime}=\partial_{n} z-m \partial_{n} \circ \partial_{n+1} t=0$, so $z^{\prime} \in \operatorname{Ker}_{n} \cap F_{n}$. Furthermore, $z-z^{\prime}=m \partial_{n+1} t=\partial_{n+1}(m t) \in \operatorname{Im} \partial_{n+1}$. As for condition (ii), suppose that $z^{\prime} \in \operatorname{Ker} \partial_{n} \cap F_{n}$ and $z^{\prime}=\partial_{n+1} c$ for some $c \in E_{n+1}$. Write $c=m t+c^{\prime}$, with $t$ not involved in $c^{\prime}$. Then $\partial_{n+1} c=m s-m r+\partial_{n+1} c^{\prime}$. Since $\partial_{n+1} c=z^{\prime} \in \operatorname{Ker}_{n} \cap F_{n}$, $\partial_{n+1} c$ does not involve $s$. Therefore, $m=0$ and $c \in F_{n+1}$.

In dimension $n-1$, condition (i) follows from the fact that $\operatorname{Ker}_{n-1} \cap F_{n-1}=$ Ker $\partial_{n-1}$. Suppose now that $z^{\prime} \in \operatorname{Ker}_{n-1} \cap F_{n-1}$ and $z^{\prime}=\partial_{n} c$ for some $c \in E_{n}$. Write $c=m s+c^{\prime}$, with $c^{\prime} \in F_{n}$. Then $c-m \partial_{n+1} t=c^{\prime}+m r \in F_{n}$, and $\partial_{n}\left(c^{\prime}+m r\right)=$ $\partial_{n} c-m \partial_{n} \circ \partial_{n+1} t=\partial_{n} c=z^{\prime}$. Thus condition (ii) holds as well.

Remark 2.2. In Section 4 we will use the special version of Theorem 2.1 where the chain complex $E_{*}$ is the chain complex of a closed simplicial complex $L$ and $F_{*}$ is the subchain complex of $E_{*}$ associated to a closed simplicial subcomplex $M$ of $L$ obtained by removing two appropriate simplexes. 
A chain map $f: E_{*} \longrightarrow F_{*}$ is a chain equivalence if there is a chain map $g$ : $F_{*} \longrightarrow E_{*}$ such that $g \circ f \simeq 1_{E_{*}}$ and $f \circ g \simeq 1_{F_{*}}$. Here, $f \simeq h$ means that the chain maps $f: E_{*} \longrightarrow F_{*}$ and $h: E_{*} \longrightarrow F_{*}$ are chain homotopic, i.e., there is a sequence of maps $s_{n}: E_{n} \longrightarrow F_{n+1}$ such that $f_{n}-h_{n}=\delta_{n+1} \circ s_{n}+s_{n-1} \circ \partial_{n}$ for all $n \in \mathbb{Z}$. This is an equivalence relation compatible with composition. hComp will denote the category whose objects are chain complexes and whose morphisms are homotopy classes of chain maps. Note that we have a natural functor Comp $\longrightarrow$ hComp. Similarly, we define hC్ $\mathbf{C o m p}$ and the functor $\widetilde{\mathbf{C o m p}} \longrightarrow \mathbf{h} \widetilde{\mathbf{C o m p}}$.

The following will be used quite often in this paper. If $f \simeq g$, then $H_{n}(f)=$ $H_{n}(g)$ for every $n \in \mathbb{Z}$. Also if $f: E_{*} \longrightarrow F_{*}$ is a chain equivalence, then $H_{n}(f)$ : $H_{n}\left(E_{*}\right) \longrightarrow H_{n}\left(F_{*}\right)$ is an isomorphism for every $n \in \mathbb{Z}$. Conversely, by d, Chapter II, Proposition 4.3, if $f: E_{*} \longrightarrow F_{*}$ is a chain map between free chain complexes such that $H_{n}(f): H_{n}\left(E_{*}\right) \longrightarrow H_{n}\left(F_{*}\right)$ is an isomorphism for every $n \in \mathbb{Z}$, then $f: E_{*} \longrightarrow F_{*}$ is a chain equivalence.

The method of acyclic models is useful for constructing natural chain maps and natural chain homotopies.

Definition 2.3. Let $F$ be a functor from a category $\mathbf{C}$ into the category of $R$ modules and let $\mathbf{M}$ be a set of models for $\mathbf{C}$ (i.e. a subset of $\mathrm{ObjC}$ ). We say that $F$ is free with basis in $\mathbf{M}$ if $F C$ is a free $R$-module for every $C \in \mathrm{Obj} \mathbf{C}$ and there are indexed families $\left(M_{j}\right)_{j \in J}$ of models in $\mathbf{M}$ and of elements $\left(x_{j}\right)_{j \in J}$ with $x_{j} \in F M_{j}$ such that, for every $C \in \mathrm{ObjC}$, the set $\left\{F(\sigma)\left(x_{j}\right): j \in J, \sigma: M_{j} \longrightarrow C \in \operatorname{Mor} \mathbf{C}\right\}$ is a basis of $F C$. In this situation, we call $x$ a basis of $F$ in $\mathbf{M}$.

Let $E: \mathbf{C} \longrightarrow \mathbf{C o m p}$ be a functor. We say that $E$ is free with basis in $\mathbf{M}$ if $E_{n}$ is free with basis in $\mathbf{M}$ for each $n \in \mathbb{Z}$. We say that $E$ is nonnegative if $E C$ is a nonnegative chain complex for all $C \in \mathrm{ObjC} ; E$ is acyclic on a set of models $\mathbf{M}$ for $\mathbf{C}$ if $E M$ is an acyclic chain complex for every $M \in \mathbf{M}$. A natural transformation between functors $E, F: \mathbf{C} \longrightarrow \mathbf{C o m p}$ is called a natural chain map.

An augmented natural chain map $\tau: E \longrightarrow F$ is by definition a natural transformation $\tau: E \longrightarrow F$ between two functors $E, F: \mathbf{C} \longrightarrow \widetilde{\mathbf{C o m p}}$. Note that in this case, for every $C \in \mathrm{Obj} \mathbf{C}$, the map $\left(\tau_{\mathbf{C}}\right)_{-1}: E_{-1} C \longrightarrow F_{-1} C$ is the identity $1_{R}$. An augmented natural chain map $\tau: E \longrightarrow F$ is a natural chain equivalence if, for every $C \in \mathrm{ObjC}$, the chain map $\left(\tau_{C}\right)_{*}: E_{*} C \longrightarrow F_{*} C$ is a chain equivalence of chain complexes which is natural with respect to the morphisms of $\mathbf{C}$. The notion of two natural chain maps $\tau, \rho: E \longrightarrow F$ being naturally chain homotopic is defined in a similar way.

Theorem 2.4 (Acyclic models in $\widetilde{\mathbf{C o m p}}$ ). Let $\mathbf{C}$ be a category with a set of models $\mathbf{M}$ and let $E, F: \mathbf{C} \longrightarrow \widetilde{\mathbf{C}} \mathbf{o m p}$ be functors. Then the following hold:

(1) If $F$ is free with basis in $\mathbf{M}$ and if $E$ is acyclic on $\mathbf{M}$, then there exists an augmented natural chain map $\tau: F \longrightarrow E$, and any two are naturally chain homotopic.

(2) If both $E$ and $F$ are free with basis in $\mathbf{M}$ and acyclic on $\mathbf{M}$, then every augmented natural chain map $\tau: F \longrightarrow E$ is a natural chain equivalence.

The proof of the theorem above follows from d], Chapter VI, Propositions 11.2 and 11.7, which is the acyclic models theorem in Comp. Indeed, we get (1) by observing that the identity $F_{-1} C \longrightarrow E_{-1} C$ is the same as $H_{0}(F C) \longrightarrow H_{0}(E C)$ for every $C \in \mathrm{ObjC}$. On the other hand, (2) follows from (1) by also considering 
a natural chain map $\rho: E \longrightarrow F$ and the compositions $\tau \circ \rho: E \longrightarrow E$ and $\rho \circ \tau: F \longrightarrow F$.

\section{O-Minimal homology}

In this section we will define what is an o-minimal homology theory. This definition is from $\mathrm{Wo}$.

Below, we will denote by DTOP (resp., DCTOP) the category of definable sets (resp., definably compact sets) with continuous definable maps. By DTOPP we denote the category of pairs from DTOP, that is, the objects of DTOPP are pairs $(X, A)$ with $A \subseteq X$ in DTOP and $f:(X, A) \longrightarrow(Y, B)$ is a morphism in DTOPP if $f: X \longrightarrow Y$ is a morphism in DTOP such that $f(A) \subseteq B$. In the same way we define the category of pairs DCTOPP from DCTOP.

Below $R$ is a ring with unit, $C$ is either DTOPP or DCTOPP and the functor $G: C \longrightarrow C$ is the functor that sends $(X, A) \in \operatorname{Obj} C$ into $(A, \emptyset) \in \operatorname{Obj} C$ and sends $f:(X, A) \longrightarrow(Y, B) \in \operatorname{Mor} C$ into $f_{\mid A}:(A, \emptyset) \longrightarrow(B, \emptyset) \in \operatorname{Mor} C$.

Definition 3.1. An o-minimal homology $\left(H_{*}, d_{*}\right)$ on $C$ is a sequence $\left(H_{n}\right)_{n \in \mathbb{Z}}$ of covariant functors from $C$ into the category of $R$-modules together with a sequence $\left(d_{n}\right)_{n \in \mathbb{Z}}$ of natural transformations $d_{n}: H_{n} \longrightarrow H_{n-1} \circ G$ such that the following axioms hold.

Homotopy Axiom. If $f, g:(X, A) \longrightarrow(Y, B) \in \operatorname{Mor} C$ and there is a definable homotopy in $C$ between $f$ and $g$, then

$$
H_{n}(f)=H_{n}(g): H_{n}(X, A) \longrightarrow H_{n}(Y, B)
$$

for all $n \in \mathbb{Z}$.

Exactness Axiom. For $(X, A) \in \operatorname{ObjC}$, if $i:(A, \emptyset) \longrightarrow(X, \emptyset)$ and $j:(X, \emptyset) \longrightarrow$ $(X, A)$ are the inclusions, then there exists a natural exact sequence

$$
\cdots \longrightarrow H_{n}(A, \emptyset) \stackrel{H_{n}(i)}{\rightarrow} H_{n}(X, \emptyset) \stackrel{H_{n}(j)}{\rightarrow} H_{n}(X, A) \stackrel{d_{n}}{\rightarrow} H_{n-1}(A, \emptyset) \longrightarrow \cdots
$$

(where the $d_{n}$ 's are induced by the natural transformations above).

Excision Axiom. For every $(X, A) \in \operatorname{Obj} C$ and every definable open subset $U$ of $X$ such that $\bar{U} \subseteq \AA$ and $(X-U, A-U) \in \operatorname{Obj} C$, the inclusion $(X-U, A-U) \longrightarrow$ $(X, A)$ induces isomorphisms

$$
H_{n}(X-U, A-U) \longrightarrow H_{n}(X, A)
$$

for all $n \in \mathbb{Z}$.

Dimension Axiom. If $X$ is a one point set, then $H_{n}(X, \emptyset)=0$ for all $n \neq 0$. The $R$-module $H_{0}(X, \emptyset)$ is called the coefficient $R$-module.

These axioms are the analogues of the classical Eilenberg-Steenrod axioms for homology functors (see $\mathrm{dp}, 4.1$ and 4.2). We therefore call them the o-minimal Eilenberg-Steenrod axioms.

We will write $X \in \operatorname{Obj} C$ for $(X, \emptyset) \in \operatorname{Obj} C, f: X \longrightarrow Y \in \operatorname{Mor} C$ for $f$ : $(X, \emptyset) \longrightarrow(Y, \emptyset) \in \operatorname{Mor} C$ and $H_{n}(X)$ for $H_{n}(X, \emptyset)$. Moreover, if $Q$ is the coefficient $R$-module of $\left(H_{*}, d_{*}\right)$, we will write $H_{n}(X, A ; Q)$ instead of just $H_{n}(X, A)$, and the notation $H_{n}(X, A)$ will be used for $H_{n}(X, A ; \mathbb{Z})$.

Two o-minimal homologies $\left(H_{*}, d_{*}\right)$ and $\left(H_{*}^{\prime}, d_{*}^{\prime}\right)$ on $C$ with coefficient $R$-module $Q$ are isomorphic if there is a sequence $\left(\tau_{n}\right)_{n \in \mathbb{Z}}$ of natural equivalences $\tau_{n}: H_{n} \longrightarrow$ 
$H_{n}^{\prime}$ such that

$$
\begin{array}{ccc}
H_{n+1}(X, A ; Q) & \stackrel{d_{n+1}}{\longrightarrow} & H_{n}(A ; Q) \\
\downarrow^{\tau_{n+1}} & & \downarrow^{\tau_{n}} \\
H_{n+1}^{\prime}(X, A ; Q) & \stackrel{d_{n+1}^{\prime}}{\longrightarrow} & H_{n}^{\prime}(A ; Q)
\end{array}
$$

commutes for all $(X, A) \in \operatorname{Obj} C$ and for all $n \in \mathbb{Z}$.

\section{O-Minimal Simplicial HOMOLOGY}

Here we describe how the construction of the o-minimal simplicial homology with coefficents in $\mathbb{Z}$ is done in Wo.

We start with the definable quasi-stratified triangulation theorem. Note that as in vdd, Chapter VIII, the simplicial complexes considered below are not necessarily closed as in the standard case.

Suppose that $S_{1}, \ldots, S_{k} \subseteq S \subseteq N^{m}$ are definable sets. A definable triangulation in $N^{m}$ of $S$ compatible with $S_{1}, \ldots, S_{k}$ is a pair $(\Phi, K)$ consisting of a simplicial complex $K$ in $N^{m}$ and a definable homeomorphism $\Phi: S \longrightarrow|K|$ such that each $S_{i}$ is a union of elements of $\Phi^{-1}(K)$. Here, $|K|$ is the realization of the simplicial complex $K$. We say that $(\Phi, K)$ is a stratified definable triangulation of $S$ compatible with $S_{1}, \ldots, S_{k}$ if: $m=0$ or $m>0$ and there is a stratified definable triangulation $(\Psi, L)$ of $\pi(S)$ compatible with $\pi\left(S_{1}\right), \ldots, \pi\left(S_{k}\right)$ (where $\pi: N^{m} \longrightarrow N^{m-1}$ is the projection onto the first $m-1$ coordinates) such that $\pi_{\mid \operatorname{Vert}(K)}: K \longrightarrow L$ is a simplicial map and the diagram

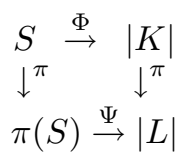

commutes. We say that $(\Phi, K)$ is a quasi-stratified definable triangulation of $S$ compatible with $S_{1}, \ldots, S_{k}$ if there is a linear bijection $\alpha: N^{m} \longrightarrow N^{m}$ such that $\left(\alpha \Phi \alpha^{-1}, \alpha K\right)$ is a stratified definable triangulation of $\alpha(S)$ compatible with $\alpha\left(S_{1}\right), \ldots, \alpha\left(S_{k}\right)$.

Theorem 4.1 (Definable quasi-stratified triangulation). Suppose that $S_{1}, \ldots, S_{k} \subseteq$ $S \subseteq N^{m}$ are definable sets. Then there is a definable triangulation of $S$ compatible with $S_{1}, \ldots, S_{k}$. Moreover, if $S$ is bounded, then there is a quasi-stratified definable triangulation of $S$ compatible with $S_{1}, \ldots, S_{k}$.

The first part of this theorem is the definable triangulation theorem, and it appears in vdd, Chapter 8 (2.9); its semi-algebraic analogue appears in dk. A careful analysis of the proof of vdd, Chapter 8 (2.9), shows that if $S$ is bounded definable set, then there is a quasi-stratified definable triangulation of $S$ compatible with $S_{1}, \ldots, S_{k}$.

We are now ready to describe the (relative) o-minimal simplicial chain complex which is defined, in [Wo, in exactly the same way as its classical analogue.

Let $K$ be a closed simplicial complex in $N^{n}$. The o-minimal simplicial chain complex $\left(C_{*}(K), \partial_{*}\right)$ is defined in the following way. For each $l \in \mathbb{N}$, we set $C_{l}(K)=$ $A_{l}(K) / A_{l}^{\prime}(K)$ where $A_{l}(K)$ is the free abelian group generated by the set of $(l+1)$ tuples $\left(v_{0}, \ldots, v_{l}\right)$ of vertices of $K$ such that $v_{0}, \ldots, v_{l}$ span an $l$-simplex in $K$, and $A_{l}^{\prime}(K)$ is the subgroup generated by the elements of the form $\left(v_{0}, \ldots, v_{l}\right)-$ $\operatorname{sgn}(\alpha)\left(v_{\alpha(0)}, \ldots, v_{\alpha(l)}\right)$ where $\alpha$ is a permutation of $\{0, \ldots, l\}$ and $v_{0}, \ldots, v_{l}$ spans 
an $l$-simplex in $K$. For $l \in \mathbb{N}$, the homomorphism $\partial_{l}: C_{l}(K) \longrightarrow C_{l-1}(K)$ is the homomorphism induced by the homomorphism

$$
\left(v_{0}, \ldots, v_{l}\right) \mapsto \sum_{i=0}^{l}(-1)^{i}\left(v_{0}, \ldots, \widehat{v}_{i}, \ldots, v_{l}\right): A_{l}(K) \longrightarrow A_{l-1}(K)
$$

where the hat on top of $v_{i}$ means that the vertice $v_{i}$ is omitted.

If we define $\widetilde{\partial}_{0}: C_{0}(K) \longrightarrow \mathbb{Z}$ by $\widetilde{\partial}_{0}\left(\sum \alpha_{i} v_{i}\right)=\sum \alpha_{i}$, we obtain an augmentation $\left(\widetilde{C}_{*}(K), \widetilde{\partial}_{*}\right)$ of $\left(C_{*}(K), \partial_{*}\right)$.

Note that each $\widetilde{C}_{l}(K)$ is a free abelian group, and given any total order on the vertices of $K$, the set of all classes $\left\langle v_{0}, \ldots, v_{l}\right\rangle \in \widetilde{C}_{l}(K)$ such that $v_{0}<\cdots<v_{l}$ provides a basis for $\widetilde{C}_{l}(K)$.

If $\left(K, K^{\prime}\right)$ is a closed simplicial pair, i.e., $K$ and $K^{\prime}$ are closed simplicial complexes with $K^{\prime}$ a subcomplex of $K$, then $\widetilde{C}_{*}\left(K^{\prime}\right)$ is a subcomplex of $\widetilde{C}_{*}(K)$, and we define the relative o-minimal simplicial chain complex $\left(C_{*}\left(K, K^{\prime}\right), \partial_{*}\right)$ by setting $C_{*}\left(K, K^{\prime}\right)=\widetilde{C}_{*}(K) / \widetilde{C}_{*}\left(K^{\prime}\right)$. Note that $C_{*}(K) \simeq C_{*}(K, \emptyset)$. We define

$$
H_{*}\left(K, K^{\prime}\right)=H_{*}\left(\left(C_{*}\left(K, K^{\prime}\right), \partial_{*}\right)\right)=\operatorname{Ker} \partial_{*} / \operatorname{Im} \partial_{*+1} .
$$

Below we will denote by CSCP the category whose objects are closed simplicial pairs in $N^{m}$ for some $m$, and whose morphisms are the arrows $f:\left(K, K^{\prime}\right) \longrightarrow$ $\left(L, L^{\prime}\right)$ such that $f:\left(|K|,\left|K^{\prime}\right|\right) \longrightarrow\left(|L|,\left|L^{\prime}\right|\right)$ is a morphism in DTOPP. By CSC we will denote the full subcategory of CSCP such that $K \in \mathrm{ObjCSC}$ if and only if $(K, \emptyset) \in \operatorname{ObjCSCP}$ and $f: K \longrightarrow L \in$ MorCSC if and only if $f:(K, \emptyset) \longrightarrow$ $(L, \emptyset) \in$ MorCSCP.

Having already constructed the o-minimal simplicial chain complex associated to a closed simplicial pair we need to construct the chain map associated to a morphism of closed simplicial pairs, i.e., we need to prove the following result:

Theorem 4.2. There is a well defined functor from the category CSCP into $\mathbf{h} C o m p$ sending $\left(K, K^{\prime}\right) \in \mathrm{ObjCSCP}$ into $\left(C_{*}\left(K, K^{\prime}\right), \partial_{*}\right)$ and sending $f:\left(K, K^{\prime}\right) \longrightarrow$ $\left(L, L^{\prime}\right) \in \operatorname{MorCSCP}$ into $f_{\sharp}: C_{*}\left(K, K^{\prime}\right) \longrightarrow C_{*}\left(L, L^{\prime}\right)$.

In the classical case, it is at this point that the simplicial approximation theorem plays a role (see $\mathrm{dp}, 5.4)$. As we pointed out already, this approach fails in the o-minimal context because the field $(N, 0,1,<,+, \cdot)$ may be non-Archimedian. The second author handled this difficulty in Wo in the way that we will now present. Roughly, we start by proving Theorem 4.2 for compatible definable maps $f: K \longrightarrow L \in$ MorCSC (see Definition 4.3 and Lemma 4.5 below) and then use definable stratified triangulations to generalize the construction to arbitrary definable maps $f: K \longrightarrow L \in$ MorCSC.

Definition 4.3. For a closed simplicial complex $K$, let $\mathbf{A}(K)$ denote the category whose objects are closed simplicial subcomplexes of $K$ and whose morphisms are the inclusion maps.

Let $\widetilde{C}_{*}^{L}: \mathbf{A}(L) \longrightarrow \widetilde{\mathbf{C}}$ omp be the functor which sends a closed subcomplex $L^{\prime}$ of $L$ to the augmented chain complex $\widetilde{C}_{*}\left(L^{\prime}\right)$ and sends an inclusion $L^{\prime \prime} \longrightarrow L^{\prime}$ to the inclusion $\widetilde{C}_{*}\left(L^{\prime \prime}\right) \longrightarrow \widetilde{C}_{*}\left(L^{\prime}\right)$.

We say that $f: K \longrightarrow L \in$ MorCSC is compatible if, for each simplex $\tau \in K$, there is a simplex $\sigma \in L$ such that $f(|\tau|) \subseteq|\sigma|$. For each compatible $f: K \longrightarrow$ $L \in$ MorCSC, we define the functor $\mathbf{A}_{f}: \mathbf{A}(L) \longrightarrow \mathbf{A}(K)$ by $\mathbf{A}_{f}\left(L^{\prime}\right)=\{\tau \in K$ : $\left.f(|\tau|) \subseteq\left|L^{\prime}\right|\right\}$. 
Lemma 4.4. If $f: K \longrightarrow L \in$ MorCSC is a compatible definable map, then there is an augmented natural chain map $\widetilde{C}_{*}^{K} \circ \mathbf{A}_{f} \longrightarrow \widetilde{C}_{*}^{L}$, and any two are naturally chain homotopic.

Proof. The set $\mathbf{M}_{L}=\{\bar{\sigma}: \sigma \in L\}$ is a set of models for $\mathbf{A}(L)$ and clearly $\widetilde{C}_{n}^{L}$ is acyclic on $\mathbf{M}_{L}$.

Fix a total ordering of the vertices of $K$. For $n \in \mathbb{N}$, denote by $I_{n}^{K}$ the basis of $\widetilde{C}_{n}(K)$ consisting of all classes $\left\langle v_{0}, \ldots, v_{n}\right\rangle$ such that $v_{0}<\cdots<v_{n}$ and identify $\alpha \in I_{n}^{K}$ with the simplex determined by $\alpha$. For each $\alpha \in I_{n}^{K}$, let $\tau_{\alpha}$ be the unique simplex of $L$ such that $f(|\alpha|) \subseteq\left|\tau_{\alpha}\right|$. Then $\left(\bar{\tau}_{\alpha}\right)_{\alpha \in I_{n}^{K}}$ is a family of models in $\mathbf{M}_{L}$ and $\left\{\widetilde{C}_{n}^{K} \circ \mathbf{A}_{f}(\ell)\left(\bar{\tau}_{\alpha}\right): \alpha \in I_{n}^{K}\right.$ and $\ell: \bar{\tau}_{\alpha} \longrightarrow S$ an inclusion $\}$ is a basis of $\widetilde{C}_{n}^{K} \circ \mathbf{A}_{f}(S)$ for every $S \in \operatorname{Obj} \mathbf{A}(L)$. So $\widetilde{C}_{*}^{K} \circ \mathbf{A}_{f}$ is free with basis in $\mathbf{M}_{L}$. Now apply the method of acyclic models (Theorem 2.4).

Here is a consequence of Lemma 4.4

Lemma 4.5. For every compatible definable map $f: K \longrightarrow L \in$ MorCSC there exists a chain map $f_{\sharp}: \widetilde{C}_{*}(K) \longrightarrow \widetilde{C}_{*}(L)$ which is the homotopy class of chain maps $\widetilde{C}_{*}(K) \longrightarrow \widetilde{C}_{*}(L)$ determined by augmented natural chain maps $\widetilde{C}_{*}^{K} \circ \mathbf{A}_{f} \longrightarrow \widetilde{C}_{*}^{L}$ given by Lemma 4.4. Moreover, if $h: K \longrightarrow L$ and $g: L \longrightarrow M$ in MorCSC are compatible definable maps, then $(g \circ h)_{\sharp}=g_{\sharp} \circ h_{\sharp}$.

Proof. Let $\rho$ (resp., $\lambda$ ) be an augmented natural chain map $\widetilde{C}_{*}^{K} \circ \mathbf{A}_{h} \longrightarrow \widetilde{C}_{*}^{L}$ (resp., $\left.\widetilde{C}_{*}^{L} \circ \mathbf{A}_{g} \longrightarrow \widetilde{C}_{*}^{M}\right)$ given by Lemma 4.4. For each $S \in \operatorname{ObjA}(M)$ define the chain $\operatorname{map} \mu_{S}: \widetilde{C}_{*}^{K} \circ \mathbf{A}_{g \circ h}(S) \longrightarrow \widetilde{C}_{*}^{L} \circ \mathbf{A}_{g}(S)$ by setting $\mu_{S}=\lambda_{\mathbf{A}_{g}(M)}$. Then $\mu$ is an augmented natural chain map $\widetilde{C}_{*}^{K} \circ \mathbf{A}_{g \circ h} \longrightarrow \widetilde{C}_{*}^{L} \circ \mathbf{A}_{g}$, and the composition $\lambda \circ \mu$ is an augmented natural chain map $\widetilde{C}_{*}^{K} \circ \mathbf{A}_{g \circ h} \longrightarrow \widetilde{C}_{*}^{M}$. Since $\lambda_{M} \circ \rho_{L}=(\lambda \circ \mu)_{M}$ it follows that $\left[\lambda_{M}\right] \circ\left[\rho_{L}\right]=\left[(\lambda \circ \mu)_{M}\right]$. Thus $g_{\sharp} \circ h_{\sharp}=(g \circ h)_{\sharp}$.

Our next goal is to prove the following result which will allow us to define a chain map $f_{\sharp}: \widetilde{C}_{*}(K) \longrightarrow \widetilde{C}_{*}(L)$ for $f: K \longrightarrow L \in$ MorCSC as a definable map not necessarily compatible.

Lemma 4.6. Let $K$ be a closed simplicial complex and let $(\Psi, L)$ be a quasistratified triangulation of $|K|$ such that $\Psi^{-1}: L \longrightarrow K$ is compatible. Then every augmented natural chain map $\widetilde{C}_{*}^{L} \circ \mathbf{A}_{\Psi^{-1}} \longrightarrow \widetilde{C}_{*}^{K}$ is a natural chain equivalence.

Lemma 4.6 is an immediate consequence of the following result:

Lemma 4.7. Let $X$ be a nonempty, closed, bounded, convex, definable subset of $N^{n}$ and $(\Psi, L)$ a stratified triangulation of $X$. Then $\widetilde{C}_{*}(L)$ is an acyclic chain complex.

Proof of Lemma 4.6. Let $K$ be a closed simplicial complex and let $(\Psi, L)$ be a quasi-stratified triangulation of $|K|$ such that $\Psi^{-1}: L \longrightarrow K$ is compatible. As in the proof of Lemma 4.4, $\widetilde{C}_{*}^{K}$ is acyclic on $\mathbf{M}_{K}=\{\bar{\sigma}: \sigma \in K\}$, and $\widetilde{C}_{*}^{L} \circ \mathbf{A}_{\Psi^{-1}}$ and $\widetilde{C}_{*}^{K}=\widetilde{C}_{*}^{K} \circ \mathbf{A}_{1_{K}}$ are free with a basis in $\mathbf{M}_{K}$. For each $\bar{\sigma} \in \mathbf{M}_{K},\left(\Psi_{|| \bar{\sigma} \mid}, \mathbf{A}_{\Psi^{-1}}(\bar{\sigma})\right)$ is a quasi-stratified triangulation of the convex set $|\bar{\sigma}|$. By Lemma 4.7, $\widetilde{C}_{*}^{L} \circ \mathbf{A}_{\Psi^{-1}}$ is acyclic on $\mathbf{M}_{K}$. Hence, by the method of acyclic models (Theorem 2.4) we conclude that every augmented natural chain map $\widetilde{C}_{*}^{L} \circ \mathbf{A}_{\Psi^{-1}} \longrightarrow \widetilde{C}_{*}^{K}$ is a natural chain equivalence as required. 
We now go on to prove Lemma 4.7. Below, by $\pi: N^{n+1} \longrightarrow N^{n}$ we will denote the projection map onto the first $n$ coordinates.

Proof of Lemma 4.7. A pair $(K, L)$ of simplicial complexes is said to be a projective pair if for some $n \in \mathbb{N}, K$ is a nonempty, closed simplicial complex in $N^{n}, L$ is a closed simplicial complex in $N^{n+1}$, the restriction $\pi_{\mid \operatorname{Vert}(\mathrm{L})}: L \longrightarrow K$ is a simplicial map and $\pi(|L|)=|K|$.

We say that a projective pair $(K, L)$ of simplicial complexes has convex fibers if, for each $\left(x_{1}, \ldots, x_{n}\right) \in|K|$, the set $\left\{x \in N:\left(x_{1}, \ldots, x_{n}, x\right) \in|L|\right\}$ is an interval $[a, b]$ in $N$, with $-\infty<a \leq b<+\infty$.

Let $(K, L)$ be a projective pair of simplicial complexes. For each $\sigma \in K$, we let $L_{\sigma}$ denote the set $\{\beta \in L: \pi(|\beta|)=|\sigma|\}$. Each set $L_{\sigma}$ is linearly ordered by defining $\beta_{1} \prec \beta_{2}$ if, for all $x=\left(x_{1}, \ldots, x_{n+1}\right) \in \beta_{1}$ and $y=\left(y_{1}, \ldots, y_{n+1}\right) \in \beta_{2}$ with $\pi(x)=\pi(y)$, we have $x_{n+1}<y_{n+1}$. A simplex $\rho \in L$ is called thin if $\operatorname{dim} \rho=\operatorname{dim} \pi \rho$, and a simplex $\lambda \in L$ is called thick if $\operatorname{dim} \lambda=\operatorname{dim} \pi \lambda+1$.

Claim 4.8. Let $(K, L)$ be a projective pair of simplicial complexes with convex fibers, and let $\sigma \in K$. Then $L_{\sigma}$ is of the form $\left\{\alpha_{0}, \beta_{1}, \alpha_{1}, \beta_{2}, \ldots, \beta_{k}, \alpha_{k}\right\}$ for some $k \geq 0$, with $\alpha_{0} \prec \beta_{1} \prec \alpha_{1} \prec \beta_{2} \prec \cdots \prec \beta_{k} \prec \alpha_{k}$, with all $\alpha_{i}$ thin and all $\beta_{i}$ thick, and with $\alpha_{i-1}$ and $\alpha_{i}$ faces of $\beta_{i}$ for $0<i \leq k$.

Proof. The set $L_{\sigma}$ is bounded above and below by the thin simplices since $L$ is closed. The rest of the claim can be deduced from the fact that: (i) if $\alpha^{\prime}, \alpha^{\prime \prime} \in L_{\sigma}$ are thin and $\alpha^{\prime} \prec \alpha^{\prime \prime}$, then there exists a thick $\beta \in L_{\sigma}$ with $\alpha^{\prime} \prec \beta \prec \alpha^{\prime \prime}$; (ii) if $\beta^{\prime}, \beta^{\prime \prime} \in L_{\sigma}$ are thick and $\beta^{\prime} \prec \beta^{\prime \prime}$, then there exists a thin $\alpha \in L_{\sigma}$ with $\beta^{\prime} \prec \alpha \prec \beta^{\prime \prime}$.

Claim 4.9. Let $(K, L)$ be a projective pair of simplicial complexes with convex fibers and suppose that $\sigma \in K$ is such that $\bar{\sigma}=K$. Then $\widetilde{C}_{*}(L)$ is acyclic.

Proof. This is proved by induction on the number of simplices of $L$. The number of simplices of $K$ is $2^{\operatorname{dim} \sigma+1}-1$. Since by Claim 4.8 the cardinality of $L_{\tau}$ is odd for each $\tau \in K$, it follows that $L$ has an odd number of simplices.

In the base case of the induction, $L$ has the same number of simplices as $K$. It follows that $L=\bar{\alpha}$ for some $\alpha \in L$ with $\operatorname{dim} \alpha=\operatorname{dim} \sigma$. In this case, $\widetilde{C}_{*}(L)$ is clearly acyclic.

So suppose that the number of simplices in $L$ is greater than in $K$. Then $L_{\sigma}=$ $\left\{\alpha_{0}, \beta_{1}, \alpha_{1}, \beta_{2}, \ldots, \beta_{k}, \alpha_{k}\right\}$ as in Claim 4.8. We consider two cases.

Case (1): $k>0$.

Let $M=L-\left\{\alpha_{k}, \beta_{k}\right\}$. First, note that $(K, M)$ is a projective pair with convex fibers. Since $M$ has two fewer simplices than $L$, it follows from the induction hypothesis that $\widetilde{C}_{*}(M)$ is acyclic. Second, note that $\beta_{k}$ is the only simplex $\beta \in L$ such that $\alpha_{k} \prec \beta$. By the theorem on removing cells (Theorem 2.1), $\widetilde{C}_{*}(M)$ is an adequate subcomplex of $\widetilde{C}_{*}(L)$, and hence $\widetilde{C}_{*}(L)$ is also acyclic.

Case (2): $k=0$, that is $L_{\sigma}=\{\alpha\}$, where $\alpha$ is a thin $\operatorname{simplex}$ of $L$ with $\operatorname{dim} \alpha=$ $\operatorname{dim} \sigma$.

Note that: (i) $\bar{\alpha}$ is a subcomplex of $L$; (ii) $\beta$ is thin for each $\beta \in \bar{\alpha}$; and for each $\tau \in \bar{\sigma}$, there exists a unique $\beta \in \bar{\alpha}$ such that $\beta \in L_{\tau}$. Let $\tau$ be a simplex in $K$ of maximal dimension such that $L_{\tau}$ contains more than one simplex. By Claim 4.8, 
$L_{\tau}$ is of the form $\left\{\rho_{0}, \lambda_{1}, \rho_{1}, \lambda_{2}, \ldots, \lambda_{l}, \rho_{l}\right\}$ for some $l>0$. Let $i \in\{0, \ldots, l\}$ be the unique index such that $\rho_{i} \in \bar{\alpha}$. Note that for all simplices $\beta \in L-L_{\tau}, \overline{|\beta|} \cap\left|L_{\tau}\right| \neq \emptyset$ implies that $\tau \prec \pi \beta$ and $\pi \beta \prec \sigma$ or $\pi \beta=\sigma$, and hence $\overline{|\beta|} \cap\left|L_{\tau}\right|=\rho_{i}$.

If $i=0$, then $\lambda_{l}$ is the only simplex $\beta \in L$ such that $\rho_{l} \prec \beta$. Let $M=L-\left\{\rho_{l}, \lambda_{l}\right\}$. Then $(K, M)$ is a projective pair with convex fibers and, once again, since $M$ has two fewer simplices than $L, \widetilde{C}_{*}(M)$ is acyclic by induction. By the theorem on removing cells (Theorem 2.1), $\widetilde{C}_{*}(M)$ is an adequate subcomplex of $\widetilde{C}_{*}(L)$, and hence $\widetilde{C}_{*}(L)$ is also acyclic.

The case $i>0$ is similar. Simply let $M=L-\left\{\rho_{0}, \lambda_{1}\right\}$.

We are now ready to finish the proof of Lemma 4.7. We use induction on $n$. The case $n=0$ is trivial. So suppose that $n>0$. Then $(\Psi, L)$ is a lifting of a stratified triangulation $(\Phi, K)$ of $\pi(X)$. It follows that $(K, L)$ is a projective pair and, in particular, it follows from the commutativity property of liftings and the convexity of $X$ that $(K, L)$ has convex fibers. Note that $\pi_{L}=\pi_{|| L \mid}: L \longrightarrow K$ is compatible, so by Lemma 4.4 there is a natural chain map $\widetilde{C}_{*}^{L} \circ \mathbf{A}_{\pi_{L}} \longrightarrow \widetilde{C}_{*}^{K}$. By induction, $\widetilde{C}_{*}(K)$ is acyclic. Thus it suffices to show that the natural chain map $\widetilde{C}_{*}^{L} \circ \mathbf{A}_{\pi_{L}} \longrightarrow \widetilde{C}_{*}^{K}$ is a natural chain equivalence.

As in the proof of Lemma 4.4, $\widetilde{C}_{*}^{K}$ is acyclic on $\mathbf{M}_{K}=\{\bar{\sigma}: \sigma \in K\}$, and $\widetilde{C}_{*}^{L} \circ \mathbf{A}_{\pi_{L}}$ and $\widetilde{C}_{*}^{K}=\widetilde{C}_{*}^{K} \circ \mathbf{A}_{1_{K}}$ are free with a basis in $\mathbf{M}_{K}$. By Claim 4.9, $\widetilde{C}_{*}\left(\pi_{L}^{-1}(|\bar{\sigma}|)\right)$ is an acyclic chain complex for each $\sigma \in K$, i.e., $\widetilde{C}_{*}^{L} \circ \mathbf{A}_{\pi_{L}}$ is acyclic on $\mathbf{M}_{K}$. The method of acyclic models (Theorem 2.4) completes the proof.

Finally we are ready to prove Theorem 4.2 .

Proof of Theorem 4.2. Suppose $f: K \longrightarrow L \in$ MorCSC is a definable map not necessarily compatible. Let $(\Phi, M)$ be a quasi-stratified triangulation of $|K|$ such that $\Phi^{-1}: M \longrightarrow K$ and $f \circ \Phi^{-1}: M \longrightarrow L$ are compatible. Define $f_{\sharp}^{(\Phi, M)}$ to be $\left(f \circ \Phi^{-1}\right)_{\sharp} \circ\left(\Phi^{-1}\right)_{\sharp}^{-1}: \widetilde{C}_{*}(K) \longrightarrow \widetilde{C}_{*}(L)$.

Claim 4.10. Suppose that $f: K \longrightarrow L \in \operatorname{MorCSC}$ and $(\Phi, M)$ is as above. Let $(\Psi, N)$ be a quasi-stratified triangulation of $|K|$ such that $\Phi \circ \Psi^{-1}: N \longrightarrow M$ is compatible. Then $f_{\sharp}^{(\Phi, M)}=f_{\sharp}^{(\Psi, N)}$.

Proof.

$$
\begin{aligned}
& f_{\sharp}^{(\Psi, N)}=\left(f \circ \Psi^{-1}\right)_{\sharp} \circ\left(\left(\Psi^{-1}\right)_{\sharp}\right)^{-1} \\
& =\left(f \circ \Phi^{-1} \circ \Phi \circ \Psi^{-1}\right)_{\sharp} \circ\left(\left(\Phi^{-1} \circ \Phi \circ \Psi^{-1}\right)_{\sharp}\right)^{-1} \\
& =\left(f \circ \Phi^{-1}\right)_{\sharp} \circ\left(\Phi \circ \Psi^{-1}\right)_{\sharp} \circ\left(\left(\Phi \circ \Psi^{-1}\right)_{\sharp}\right)^{-1} \circ\left(\left(\Phi^{-1}\right)_{\sharp}\right)^{-1} \\
& =f_{\sharp}^{(\Phi, M)} .
\end{aligned}
$$

It follows easily from Claim 4.10 that $f_{\sharp}^{(\Phi, M)}$ is independent of the triangulation. In light of this, we will use the simpler notation $f_{\sharp}$ to denote the homotopy class of chain maps determined by $f$.

Claim 4.11. Let $f: K \longrightarrow K^{\prime}$ and $g: K^{\prime} \longrightarrow K^{\prime \prime}$ be morphisms of CSC. Then $(g \circ f)_{\sharp}=g_{\sharp} \circ f_{\sharp}$.

Proof. Let $(\Phi, M)$ be an appropriate triangulation of $\left|K^{\prime}\right|$ such that $g_{\sharp}=g_{\sharp}^{(\Phi, M)}$. By the definable triangulation theorem, there is a quasi-stratified triangulation 
$(\Psi, N)$ of $|K|$ such that $\Phi \circ f \circ \Psi^{-1}: N \longrightarrow M$ is compatible. We use the fact that $\sharp$ distributes over a composition of compatible maps:

$$
\begin{aligned}
& (g \circ f)_{\sharp}=(g \circ f)_{\sharp}^{(\Psi, N)} \\
& =\left(g \circ f \circ \Psi^{-1}\right)_{\sharp} \circ\left(\left(\Psi^{-1}\right)_{\sharp}\right)^{-1} \\
& =\left(g \circ \Phi^{-1} \circ \Phi \circ f \circ \Psi^{-1}\right)_{\sharp} \circ\left(\left(\Psi^{-1}\right)_{\sharp}\right)^{-1} \\
& =\left(g \circ \Phi^{-1}\right)_{\sharp} \circ\left(\left(\Phi^{-1}\right)_{\sharp}\right)^{-1} \circ\left(\Phi^{-1}\right)_{\sharp} \circ\left(\Phi \circ f \circ \Psi^{-1}\right)_{\sharp} \circ\left(\left(\Psi^{-1}\right)_{\sharp}\right)^{-1} \\
& =g_{\sharp}^{(\Phi, M)} \circ f_{\sharp}^{(\Psi, N)}=g_{\sharp} \circ f_{\sharp} .
\end{aligned}
$$

For any $f:\left(K, K^{\prime}\right) \longrightarrow\left(L, L^{\prime}\right)$, there is a representative $\tau_{K}$ of the homotopy class $f_{\sharp}$ such that $\tau_{K}\left(\widetilde{C}_{*}\left(K^{\prime}\right)\right) \subseteq \widetilde{C}_{*}\left(L^{\prime}\right)$. Therefore $f_{\sharp}$ induces a homotopy class of chain maps $C_{*}\left(K, K^{\prime}\right) \longrightarrow C_{*}\left(L, L^{\prime}\right)$. Thus we have established one of the main results of this section, namely Theorem 4.2 .

With Theorem 4.2 available, the o-minimal simplicial homology is defined in $\mathrm{Wo}$ in the following way.

Definition 4.12. For $(X, A) \in \operatorname{ObjDCTOPP}$, let $T(X, A)$ denote the set of all definable triangulations $(\Phi, K)$ of $X$ which respects $A$, and for each $(\Phi, K) \in$ $T(X, A)$, let $K^{\prime}$ denote the closed subcomplex of $K$ such that $\Phi(A)=\left|K^{\prime}\right|$. The $n$-th simplicial homology group $H_{n}(X, A)$ is defined to be the subgroup of $\prod_{(\Phi, K) \in T(X, A)} H_{n}\left(K, K^{\prime}\right)$ consisting of all elements $\alpha$ such that, for all $\left(\Phi_{1}, K_{1}\right)$, $\left(\Phi_{2}, K_{2}\right) \in T(X, A)$, we have $\alpha_{\left(\Phi_{2}, K_{2}\right)}=H_{n}\left(\Phi_{2} \circ \Phi_{1}^{-1}\right)\left(\alpha_{\left(\Phi_{1}, K_{1}\right)}\right)$.

Given $f:(X, A) \longrightarrow(Y, B) \in$ MorDCTOPP we define the $n$-th induced homomorphism $H_{n}(f): H_{n}(X, A) \longrightarrow H_{n}(Y, B)$ such that if $H_{n}(f)(\alpha)=\beta$, then for all $(\Phi, K) \in T(X, A)$ and $(\Psi, L) \in T(Y, B)$, we have

$$
\beta_{(\Psi, L)}=H_{n}\left(\left(\Psi \circ f \circ \Phi^{-1}\right)_{\sharp}\right)\left(\alpha_{(\Phi, K)}\right) .
$$

The verification of the Eilenberg-Steenrod axioms is now easy. In fact, the arguments are similar to their analogues for the classical topological simplicial homology.

Theorem 4.13. There is an o-minimal homology $\left(H_{*}, d_{*}\right)$ on DCTOPP with coefficients in $\mathbb{Z}$ such that if $(X, A) \in \operatorname{ObjDCTOPP},(\Phi, K)$ is a definable triangulation of $X$ which respects $A$ and $K^{\prime}$ is the subcomplex of $K$ such that $\left|K^{\prime}\right|=\Phi(A)$, then we have isomorphisms $\pi_{n}^{(\Phi, K)}: H_{n}(X, A) \longrightarrow H_{n}\left(K, K^{\prime}\right)$ for all $n \in \mathbb{Z}$. Also, if $f:(X, A) \longrightarrow(Y, B) \in$ MorDCTOPP and $(\Psi, L)$ is a definable triangulation of $Y$ respecting $B$ and such that $\left|L^{\prime}\right|=\Psi(B)$, then the diagram

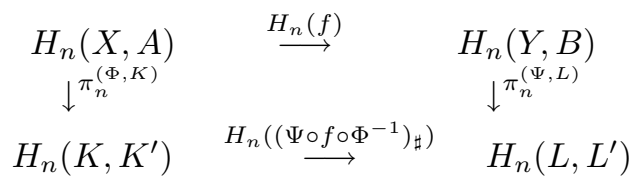

is commutative.

Proof. By the above, it follows easily that each $H_{n}$ defines a functor from DCTOPP into the category of abelian groups. We now verify the o-minimal EilenbergSteenrod axioms for the sequence of functors $H_{*}$ we have just defined.

Proof of the homotopy axiom. If $F:(X \times[a, b], A \times[a, b]) \longrightarrow(Y, B)$ is a definable homotopy in DCTOPP between $f_{a}, f_{b}:(X, A) \longrightarrow(Y, B) \in$ MorDCTOPP, then $f_{c}=F \circ g_{c}$ where for $c=a, b, g_{c}:(X, A) \longrightarrow(X \times[a, b], A \times[a, b]) \in$ MorDCTOPP 
is given by $g_{c}(x)=(x, c)$. So to prove the homotopy axiom, it is clearly enough to show that $H_{*}\left(g_{a}\right)=H_{*}\left(g_{b}\right)$. Let $\left(K, K^{\prime}\right) \in T(X, A)$. Then, we have a canonical $\left(M, M^{\prime}\right) \in T(X \times[a, b], A \times[a, b])$ associated to $\left(K, K^{\prime}\right)$ such that the induced morphisms $g_{c}: K \longrightarrow M(c=a, b)$ and $\pi: M \longrightarrow K$ (induced by the projection $\pi: X \times[a, b] \longrightarrow X)$ in MorCSC are compatible. Since the morphisms $g_{c}: K \longrightarrow$ $M$ are compatible, by Lemma 4.4, they induce augmented natural chain maps $\tau^{c}: \widetilde{C}_{*}^{K} \circ \mathbf{A}_{g_{c}} \longrightarrow \widetilde{C}_{*}^{M}$, which induce chain maps $\left(g_{c}\right)_{\sharp}: C_{*}\left(K, K^{\prime}\right) \longrightarrow C_{*}\left(M, M^{\prime}\right)$. Since, by definition $H_{*}\left(g_{c}\right)=H_{*}\left(\left(g_{c}\right)_{\sharp}\right)$, it is enough to show that $\left(g_{a}\right)_{\sharp}$ and $\left(g_{b}\right)_{\sharp}$ are chain homotopic.

Let $\mathbf{B}$ be the subcategory of $\mathbf{A}(M)$ consisting of subcomplexes of $M$ of the form $\mathbf{A}_{\pi}(S)$, where $S$ is a closed subcomplex of $K$. It is easy to see that: (1) the set $\mathbf{M}=\left\{\mathbf{A}_{\pi}(\bar{\sigma}): \sigma \in K\right\}$ is a set of models for $\mathbf{B} ;(2)$ each $\tau^{c}$ restricts to an augmented natural chain map $\tau_{\mid \mathbf{B}}^{c}: \widetilde{C}_{*}^{K} \circ \mathbf{A}_{g_{c} \mid \mathbf{B}} \longrightarrow\left(\widetilde{C}_{*}^{M}\right)_{\mid \mathbf{B}}$ and $(3) \widetilde{C}_{*}^{K} \circ \mathbf{A}_{g_{c} \mid \mathbf{B}}$ is free with basis in $\mathbf{M}$ and $\left(\widetilde{C}_{*}^{M}\right)_{\mid \mathbf{B}}$ is acyclic on $\mathbf{M}$ (by Claim 4.9 , since $\left(\bar{\sigma}, \mathbf{A}_{\pi}(\bar{\sigma})\right.$ ) is a projective pair of simplicial complexes with convex fibers). Therefore, by the method of acyclic models (Theorem 2.4), there is a natural chain homotopy $\tau_{\mid \mathbf{B}}^{a} \simeq \tau_{\mid \mathbf{B}}^{b}$ which induces the desired chain homotopy $\left(g_{a}\right)_{\sharp} \simeq\left(g_{b}\right)_{\sharp}$.

Proof of the exactness axiom. Suppose that $(X, A) \in \operatorname{ObjDCTOPP}$ and $\left(K, K^{\prime}\right) \in$ $T(X, A)$. Then we have a short exact sequence of chain maps

$$
0 \rightarrow C_{*}\left(K^{\prime}\right) \rightarrow C_{*}(K) \rightarrow C_{*}\left(K, K^{\prime}\right) \rightarrow 0
$$

which, by basic homological algebra ([sp], Chapter 4, Section 5, Lemma 3 and Theorem 4), gives connecting homomorphisms $d_{n}^{K, K^{\prime}}: H_{n}\left(K, K^{\prime}\right) \longrightarrow H_{n-1}\left(K^{\prime}\right)$ making the induced sequence

$$
\cdots \longrightarrow H_{n}\left(K^{\prime}, \emptyset\right) \rightarrow H_{n}(K, \emptyset) \rightarrow H_{n}\left(K, K^{\prime}\right) \stackrel{d_{n}^{K, K^{\prime}}}{\rightarrow} H_{n-1}\left(K^{\prime}, \emptyset\right) \longrightarrow \cdots
$$

exact. Since these connecting homomorphisms are induced by natural transformations in the category of short exact sequences of chain maps ([sp], Chapter 4, Section 5, Lemma 3 and Theorem 4), they determine natural transformations $d_{n}: H_{n} \longrightarrow H_{n-1} \circ G$ satisfying the exactness axiom by requiring that the diagram

$$
\begin{array}{ccc}
H_{n}(X, A) & \stackrel{d_{n}^{X, A}}{\longrightarrow} & H_{n-1}(A) \\
\downarrow^{\pi^{\left(K, K^{\prime}\right)}} & & \downarrow^{\pi^{\left(K^{\prime}, \emptyset\right)}} \\
H_{n}\left(K, K^{\prime}\right) & \stackrel{d_{n}^{K, K^{\prime}}}{\longrightarrow} & H_{n-1}\left(K^{\prime}\right)
\end{array}
$$

commutes.

Proof of the excision axiom. Let $(X, A) \in$ ObjDCTOPP and let $U \in$ ObjDTOP be an open subset of $X$ such that $\bar{U} \subseteq \AA$. Let $(K, \Phi)$ be a definable triangulation of $X$ compatible with $A, X-A, U$ and $X-U$, and let $K^{\prime}$ and $K^{\prime \prime}$ be the simplicial subcomplexes of $K$ such that $A=\left|K^{\prime}\right|$ and $U=\left|K^{\prime \prime}\right|$. Let $L=K-K^{\prime \prime}$ and $L^{\prime}=K^{\prime}-K^{\prime \prime}$. Then $\left(L, \Phi_{|| L \mid}\right)$ and $\left(L^{\prime}, \Phi_{\left|L^{\prime}\right|}\right)$ are definable triangulations such that $X-U=|L|$ and $A-U=\left|L^{\prime}\right|$.

Let $G_{n}$ be the free abelian group generated by those basis elements $\left\langle v_{0}, \ldots, v_{n}\right\rangle$ of $\widetilde{C}_{n}(K)$ such that $\left(v_{0}, \ldots, v_{n}\right)$ is a simplex of $K^{\prime \prime}$. Then $G_{n}$ is a subgroup of $\widetilde{C}_{n}(K)$ and of $\widetilde{C}_{n}\left(K^{\prime}\right)$ (because $\bar{U} \subseteq \AA$ ) such that $\widetilde{C}_{n}(K)=\widetilde{C}_{n}(L) \oplus G_{n}$ and $\widetilde{C}_{n}\left(K^{\prime}\right)=$ $\widetilde{C}_{n}\left(L^{\prime}\right) \oplus G_{n}$. Thus $\frac{\widetilde{C}_{n}(K)}{\widetilde{C}_{n}\left(K^{\prime}\right)}=\frac{\widetilde{C}_{n}(L) \oplus G_{n}}{\widetilde{C}_{n}\left(L^{\prime}\right) \oplus G_{n}} \simeq \frac{\widetilde{C}_{n}(L)}{\widetilde{C}_{n}\left(L^{\prime}\right)}$, the inclusion $\left(L, L^{\prime}\right) \longrightarrow\left(K, K^{\prime}\right)$ 
induces a chain equivalence $C_{*}\left(L, L^{\prime}\right) \longrightarrow C_{*}\left(K, K^{\prime}\right)$ and hence isomorphisms in homology.

Proof of the dimension axiom. Simply note that if $K$ is a simplicial complex consisting of a single 0 -simplex, then $H_{n}(K)=0$ for all $n \neq 0$ and $H_{0}(K) \simeq \mathbb{Z}$.

\section{O-Minimal SINGULAR HOMOLOGY}

Here we include the proof, from $\mathrm{Wo}$, of the existence of the o-minimal singular homology with coefficients in $\mathbb{Z}$. We start with the o-minimal singular chain complex which is defined just as in the topological setting but replacing the topological category by the definable category.

The standard $n$-simplex $\Delta^{n}$ in DCTOP is the convex hull of the standard basis vectors $e_{0}, \ldots, e_{n}$ in $N^{n+1}$. Let the standard (-1)-simplex $\Delta^{-1}$ in DCTOP be the empty set.

Let $X \in \operatorname{ObjDTOP.~For~} n \geq-1$, we define $\widetilde{S}_{n}(X)$ to be the free abelian group on the set of definable continuous maps $\sigma: \Delta^{n} \longrightarrow X$. For $n<-1$, we set $\widetilde{S}_{n}(X)=0$. Note that $\widetilde{S}_{-1}(X)=\mathbb{Z}$. The elements of $\widetilde{S}_{n}(X)$ are called the definable $n$-chains.

For $n>0$ and $0 \leq i \leq n$ let $\epsilon_{i}^{n}: \Delta^{n-1} \longrightarrow \Delta^{n}$ be the continuous definable map given by $\epsilon_{i}^{n}\left(\sum_{j=0}^{n-1} a_{j} e_{j}\right)=\sum_{j<i} a_{j} e_{j}+\sum_{j \geq i}^{n-1} a_{j} e_{j+1}$. Let $\epsilon_{0}^{0}: \Delta^{-1} \longrightarrow \Delta^{0}$ be the unique map. We define the boundary homomorphism $\partial_{n}: \widetilde{S}_{n}(X) \longrightarrow \widetilde{S}_{n-1}(X)$ to be the trivial homomorphism for $n<0$, and for $n \geq 0, \partial_{n}$ is given on basis elements by

$$
\partial_{n}(\sigma)=\sum_{i=0}^{n}(-1)^{i} \sigma \circ \epsilon_{i}^{n}
$$

One verifies that $\partial^{2}=0$ and so $\left(\widetilde{S}_{*}(X), \partial_{*}\right)$ is a chain complex, the augmented o-minimal singular chain complex.

For $f: X \longrightarrow Y \in$ MorDTOP, we have an induced chain map $f_{\sharp}: \widetilde{S}_{*}(X) \longrightarrow$ $\widetilde{S}_{*}(Y)$ given on the basis elements of $\widetilde{S}_{*}(X)$ by $f_{\sharp}(\sigma)=f \circ \sigma$.

Given an element $\sigma=\sum_{i=1}^{k} n_{i} \sigma_{i}$ of $S_{m}(X)$ where $\sigma_{i}: \Delta^{m} \longrightarrow X$ are distinct definable continuous maps and the $n_{i}$ 's are nonzero, the support $\operatorname{Im} \sigma$ of $\sigma$ is by definition the definable subset $\bigcup\left\{\sigma_{i}\left(\Delta^{m}\right): i=1, \ldots, k\right\}$ of $X$.

If $(X, A) \in \mathrm{ObjDTOPP}$, then the relative o-minimal singular chain complex $\left(S_{*}(X, A), \partial_{*}\right)$ is the quotient chain complex $\left(\widetilde{S}_{*}(X) / \widetilde{S}_{*}(A), \partial_{*}\right)$, and the o-minimal singular chain complex $\left(S_{*}(X), \partial_{*}\right)$ is $\left(S_{*}(X, \emptyset), \partial_{*}\right)$.

For $f:(X, A) \longrightarrow(Y, B) \in$ MorDTOPP, we have an induced chain map $f_{\sharp}$ : $S_{*}(X, A) \longrightarrow S_{*}(Y, B)$. We define

$$
H_{n}(X, A)=H_{n}\left(S_{*}(X, A), \partial_{*}\right)=\operatorname{Ker} \partial_{*} / \operatorname{Im} \partial_{*+1},
$$

$H_{n}(f)=H_{n}\left(f_{\sharp}\right)$ and set $H_{n}(X)=H_{n}(X, \emptyset)$.

Having defined the o-minimal singular homology groups, it remains to show that they verify the o-minimal Eilenberg-Steenrod axioms. This will be done below and was originally done by the second author in $\mathrm{Wo}$. The argument for the o-minimal singular exactness and dimension axiom is similar to the classical case, so we refer the reader to [d], Chapter III (3.2) and (4.2). For the o-minimal singular homotopy axiom we require o-minimal versions of classical constructions. These will play an important role later in the proof of the o-minimal singular excision axiom. 
Let $X \subseteq N^{m}$ be a convex definable set and let $p \in X$. The cone construction over $p$ in $X$ is a sequence of homomorphisms $z \mapsto p . z: \widetilde{S}_{*}(X) \longrightarrow \widetilde{S}_{*+1}(X)$ defined as follows: For $n<-1, p$. is defined as the trivial homomorphism and for $n \geq-1$ and a basis element $\sigma$, we set

$$
p . \sigma\left(\sum_{i=0}^{n+1} t_{i} e_{i}\right)= \begin{cases}p & \text { if } t_{0}=1, \\ t_{0} p+\left(1-t_{0}\right) \sigma\left(\sum_{i=1}^{n+1} \frac{t_{i}}{1-t_{0}} e_{i}\right) & \text { if } t_{0} \neq 1 .\end{cases}
$$

Given objects $X, Y$ and $Z$ of DTOP, let $F(X, Y)$ denote the free abelian group on the set of all definable continuous maps from $X$ into $Y$. Given $\alpha=\sum_{i} s_{i} \alpha_{i} \in$ $F(X, Y)$ and $\beta=\sum_{j} t_{j} \beta_{j} \in F(Y, Z)$, we define the sharp operator by

$$
\beta_{\sharp} \alpha=\sum_{i, j} s_{i} t_{j}\left(\beta_{j} \circ \alpha_{i}\right) \in F(X, Z) .
$$

Note that $\sharp$ is associative, and since $\widetilde{S}_{n}(X)=F\left(\Delta^{n}, X\right)$, every $z \in \widetilde{S}_{*}(X)$ yields a chain map $z_{\sharp}: \widetilde{S}_{*}\left(\Delta^{n}\right) \longrightarrow \widetilde{S}_{*}(X)$.

Lemma 5.1. Suppose that $X \subseteq N^{m}$ is a convex definable set. Let $p \in X$ and $z \in \widetilde{S}_{n}(X)$. Then $(p . z)_{\sharp} \epsilon_{0}^{n+1}=z$ and $(p . z)_{\sharp} \epsilon_{i}^{n+1}=p \cdot\left(z_{\sharp} \epsilon_{i-1}^{n}\right)$ for $0<i \leq n$. Furthermore, $p .(\partial z)+\partial(p . z)=z$.

The proof of Lemma 5.1 is a simple computation using the definitions. For details see its classical analogue in [d, Chapter III, (4.8) and (4.9).

The cone construction is used as in the topological case to prove the following lemma. See [d], Chapter III, Proposition 4.6.

Lemma 5.2. If $X \subseteq N^{m}$ is a convex definable set, then $\widetilde{S}_{*}(X)$ is an acyclic chain complex.

Proof. It follows from Lemma [5.1 that $1_{\widetilde{S}_{n}(X)}-0_{\widetilde{S}_{n}(X)}=\partial_{n+1} \circ p_{n}+p_{n+1} \circ \partial_{n}$. Thus $p$ is a chain homotopy between the identity $1_{*}: \widetilde{S}_{*}(X) \longrightarrow \widetilde{S}_{*}(X)$ and the zero $\operatorname{map} 0_{*}: \widetilde{S}_{*}(X) \longrightarrow \widetilde{S}_{*}(X)$. Thus $H_{n}\left(1_{*}\right)=H_{n}\left(0_{*}\right)$ and $\widetilde{H}_{n}(X)=0$ for all $n \in \mathbb{Z}$.

Lemma 5.3. Consider the set of models $\mathbf{M}=\left\{\Delta^{n}: n \in \mathbb{Z}\right\}$ for DTOP. Then the functor $\widetilde{S}_{*}: \mathrm{DTOP} \longrightarrow \widetilde{\mathbf{C}} \mathbf{D} \mathbf{p}$ is acyclic on $\mathbf{M}$ and free with a basis in $\mathbf{M}$.

Proof. By Lemma 5.2, $\widetilde{S}_{*}$ is acyclic on M. Let $n \in \mathbb{Z}$. We shall construct a basis $x^{n}$ of $\widetilde{S}_{n}$ in M. Let $x^{n}$ be the identity map $1_{\Delta^{n}}: \Delta^{n} \longrightarrow \Delta^{n} \in \widetilde{S}_{n}\left(\Delta^{n}\right)$. Let $X \in$ ObjDTOP. Note that $\left\{\widetilde{S}_{n}(\sigma)\left(x^{n}\right): \sigma: \Delta^{n} \longrightarrow X \in \operatorname{MorDTOP}\right\}=\{\sigma:$ $\Delta^{n} \longrightarrow X \in$ MorDTOP $\}$, which is a basis of $\widetilde{S}_{n}(X)$ by definition.

By Lemma 5.2, the functor DTOP $\longrightarrow \widetilde{\mathbf{C}}$.omp : $X \mapsto \widetilde{S}_{*}([a, b] \times X)$ is acyclic on $\mathbf{M}$, so by Lemma 5.3 and the method of acyclic models (Theorem 2.4), there is a natural chain map from the functor $\widetilde{S}_{*}$ into this functor which is unique up to natural chain homotopy. From this and arguing as in the proof of 5.1 in [d], page 39 , we verify the o-minimal singular homotopy axiom.

We now go on to prove the o-minimal singular excision axiom. Since every definable set equipped with its topology is usually totally disconnected and never locally compact, the standard proof of the topological singular excision axiom based on repeated barycentric subdivisions and the Lebesgue number property fails. Compare with [d], Chapter III, Proposition 6.3 and 7.3. 
Definition 5.4. Let $X \in \mathrm{ObjDTOP.} \mathrm{The} \mathrm{barycentric} \mathrm{subdivision} \operatorname{Sd}_{n}: \widetilde{S}_{n}(X) \longrightarrow$ $\widetilde{S}_{n}(X)$ is defined as follows: for $n<-1, \operatorname{Sd}_{n}: \widetilde{S}_{n}(X) \longrightarrow \widetilde{S}_{n}(X)$ is the trivial homomorphism, $\operatorname{Sd}_{-1}: \widetilde{S}_{-1}(X) \longrightarrow \widetilde{S}_{-1}(X)$ is the identity and for $n \geq 0$, we set $\operatorname{Sd}_{n}(z)=z_{\sharp}\left(b_{n} . \operatorname{Sd}_{n-1} \partial 1_{\Delta^{n}}\right)$, where $b_{n}=\frac{1}{n+1}\left(e_{0}+\cdots+e_{n}\right)$ is the barycentre of $\Delta^{n}$.

Note that $\operatorname{Sd}_{0}$ is the identity and $\operatorname{Sd}_{n}$ is natural, i.e., it commutes with $f_{\sharp}$ : $\widetilde{S}_{n}(X) \longrightarrow \widetilde{S}_{n}(Y)$.

Lemma 5.5. The barycentric subdivision $\mathrm{Sd}=\left(\mathrm{Sd}_{n}\right)_{n \in \mathbb{Z}}$ is a natural chain map $\widetilde{S}_{*} \longrightarrow \widetilde{S}_{*}$ which is natural chain homotopic with the identity natural chain map $1_{*}: \widetilde{S}_{*} \longrightarrow \widetilde{S}_{*}$. Moreover, for $X \in \mathrm{ObjDTOP,} z \in \widetilde{S}_{n}(X)$ and $n \geq 0$, we have $(\mathrm{Sd} z)_{\sharp} \epsilon_{0}^{n}=\mathrm{Sd} \partial z$ and $(\mathrm{Sd} z)_{\sharp} \epsilon_{i}^{n}=0$ for $0<i \leq n$.

Proof. That Sd is a natural chain map $\widetilde{S}_{*} \longrightarrow \widetilde{S}_{*}$ is a simple computation using Lemma 5.1. We refer the reader to classical case in d], Chapter III, Proposition 6.3. The fact that $\mathrm{Sd}$ is natural chain homotopic with the identity natural chain map $1_{*}: \widetilde{S}_{*} \longrightarrow \widetilde{S}_{*}$ follows from the method of acyclic models (Theorem 2.4) since both Sd and $1_{*}$ are liftings of $1_{-1}$ and, by Lemma $5.3 \widetilde{S}_{*}$ is acyclic and free with basis in $\mathbf{M}=\left\{\Delta^{n}: n \in \mathbb{Z}\right\}$.

Now let $X \in$ ObjDTOP, $z \in \widetilde{S}_{n}(X)$ and $n \geq 0$. We want to show that $(\operatorname{Sd} z)_{\sharp} \epsilon_{0}^{n}=\operatorname{Sd} \partial z$ and $(\operatorname{Sd} z)_{\sharp} \epsilon_{i}^{n}=0$ for $0<i \leq n$. This is clear for $n=0$. So suppose that $n>0$. Then $(\operatorname{Sd} z)_{\sharp} \epsilon_{i}^{n}=z_{\sharp}\left(b_{n} \cdot \operatorname{Sd} \partial 1_{\Delta^{n}}\right)_{\sharp} \epsilon_{i}^{n}$. The latter is, by Lemma 5.1] $z_{\sharp}\left(b_{n} \cdot\left(\left(\operatorname{Sd} \partial 1_{\Delta^{n}}\right)_{\sharp} \epsilon_{i-1}^{n-1}\right)\right)$. Since $\partial 1_{\Delta^{n}} \in \widetilde{S}_{n-1}\left(\Delta^{n}\right)$, induction on $n$ yields $\left(\operatorname{Sd} \partial 1_{\Delta^{n}}\right)_{\sharp} \epsilon_{i-1}^{n-1}=0$ for $i>1$, and $\left(\operatorname{Sd} \partial 1_{\Delta^{n}}\right)_{\sharp} \epsilon_{0}^{n-1}=\operatorname{Sd} \partial^{2} 1_{\Delta^{n}}=0$. So $(\operatorname{Sd} z)_{\sharp} \epsilon_{i}^{n}=0$ for $0<i \leq n$. Since $\operatorname{Sd}$ is a natural chain map, $\operatorname{Sd} \partial z=\partial(\operatorname{Sd} z)=$ $\sum_{i=0}^{n}(-1)^{i}(\mathrm{Sd} z)_{\sharp} \epsilon_{i}^{n}=(\mathrm{Sd} z)_{\sharp} \epsilon_{0}^{n}$.

It is at this point that the classical proof of the excision axiom for topological singular homology differs from the second author proof of the o-minimal excision axiom for the o-minimal singular homology. In the classical case we apply the Lebesgue number property to the repeated barycentric subdivision operator $\mathrm{Sd}^{k}=$ $\left(\operatorname{Sd}_{n}^{k}\right)_{n \in \mathbb{Z}}: \widetilde{S}_{*}^{t o p}(X) \longrightarrow \widetilde{S}_{*}^{t o p}(X)$ where $\mathrm{Sd}^{k}$ is the composition of Sd with itself $k$ times, to prove the following lemma (see the proof of 7.3 in [d], page 44).

Lemma 5.6. Suppose that $X \in \mathrm{ObjTOP}$ and let $U$ and $V$ be open subsets of $X$ such that $X=U \cup V$. If $z \in \widetilde{S}_{n}^{\text {top }}(X)$, then there is a sufficiently large $k \in \mathbb{N}$ such that $\operatorname{Sd}_{n}^{k}(z) \in \widetilde{S}_{n}^{t o p}(U)+\widetilde{S}_{n}^{t o p}(V)$.

In the o-minimal case we use the o-minimal simplicial chain complex to define the subdivision operator which will play the role of the repeated barycentric subdivision operator.

Definition 5.7. Let $K$ be a closed simplicial complex with a fixed total ordering of its vertices. We define the natural chain map $\tau_{K}: \widetilde{C}_{*}(K) \longrightarrow \widetilde{S}_{*}(|K|)$ by setting $\tau_{K}=1_{\mathbb{Z}}: \widetilde{C}_{-1}(K) \longrightarrow \widetilde{S}_{-1}(|K|)$ and for $n \geq 0$, if $v_{0}<\cdots<v_{n}$ are vertices of an $n$-simplex of $K$ determining a basis element of $\widetilde{C}_{*}(K)$, then $\tau_{K}\left(\left\langle v_{0}, \ldots, v_{n}\right\rangle\right)=\sigma$ where $\sigma: \Delta^{n} \longrightarrow|K|$ is such that $\sigma\left(\sum_{i=0}^{n} t_{i} e_{i}\right)=\sum_{i=0}^{n} t_{i} v_{i}$. 
Definition 5.8. For $i \leq n$, the definable continuous map $\gamma_{i}^{n}: \Delta^{n} \longrightarrow \Delta^{i}$ is defined by

$$
\gamma_{i}^{n}\left(\sum_{j=0}^{n} a_{j} e_{j}\right)=\sum_{j=0}^{i}\left(a_{n-i+j}+\frac{\sum_{k=0}^{n-i-1} a_{k}}{i+1}\right) e_{j} .
$$

So $\gamma_{i}^{n}$ maps $\Delta^{n}$ to $\Delta^{i}$ by sending the first $n-i$ vertices of $\Delta^{n}$ to the barycenter of $\Delta^{i}$.

Let $(\Phi, K)$ be a definable triangulation of $\Delta^{n}$ compatible with the standard simplicial complex $E^{n}$ determined by $\Delta^{n}$. For $X \in$ ObjDTOP, we define the subdivision operator $\operatorname{Sd}_{i}^{K}: \widetilde{S}_{i}(X) \longrightarrow \widetilde{S}_{i}(X)$ where $i \leq n$ by: $\operatorname{Sd}_{-1}^{K}=1_{\mathbb{Z}}$ and for $0 \leq i \leq n$

$$
\operatorname{Sd}_{i}^{K}(z)=(\operatorname{Sd} z)_{\sharp}\left(\gamma_{i}^{n}\right)_{\sharp}\left(\Phi^{-1}\right)_{\sharp} \tau_{K} \Phi_{\sharp}\left\langle e_{n-i}, \ldots, e_{n}\right\rangle
$$

where $\Phi_{\sharp}: \widetilde{C}_{*}\left(E^{n}\right) \longrightarrow \widetilde{C}_{*}(K)$ is a chain map induced by $\Phi: \Delta^{n} \longrightarrow|K|$ (Theorem 4.2.).

Note that, if $s \in E^{n}$ is a simplex, then for each $i \in \mathbb{Z}$ we have $\Phi_{\sharp}\left(\widetilde{C}_{i}(\bar{s})\right) \subseteq$ $\widetilde{C}_{i}\left(K_{|| \bar{s} \mid}\right)$ where $\left(\Phi_{|| \bar{s} \mid}, K_{|| \bar{s} \mid}\right)$ is the definable triangulation of $|\bar{s}|$ obtained by restriction.

We now prove the analogue of Lemma 5.5 for the subdivision operator. The role of $\gamma_{i}^{n}$ in the definition of the subdivision operator is rather technical and appears in the proof of the next lemma.

Lemma 5.9. The subdivision operator $\mathrm{Sd}^{K}=\left(\operatorname{Sd}_{i}^{K}\right)_{i \leq n}$ is a natural partial chain map $\widetilde{S}_{*} \longrightarrow \widetilde{S}_{*}$ of order $n$, i.e., $\operatorname{Sd}_{i}^{K}$ is natural and $\partial \operatorname{Sd}_{i}^{K}=\operatorname{Sd}_{i-1}^{K} \partial$ for each $i \leq n$. Moreover, there is a natural partial chain homotopy between the subdivision operator $\mathrm{Sd}^{K}$ and the restriction $\left(1_{i}\right)_{i \leq n}$ of the identity natural chain map $1_{*}: \widetilde{S}_{*}(X) \longrightarrow$ $\widetilde{S}_{*}(X)$.

Proof. It is clear from the definition that $\mathrm{Sd}_{i}^{K}$ is natural. We need to show that $\operatorname{Sd}^{K}: \widetilde{S}_{*}(X) \longrightarrow \widetilde{S}_{*}(X)$ is a partial chain map of order $n$ for every $X \in$ ObjDTOP.

Let $z \in \widetilde{S}_{i}(X)$ where $i \leq n$. The case $i \leq 0$ is trivial. So suppose that $i>0$. Then by the definitions and the fact that we are dealing with chain maps we get

$$
\partial\left(\operatorname{Sd}_{i}^{K} z\right)=\sum_{j=0}^{i}(-1)^{j}(\operatorname{Sd} z)_{\sharp}\left(\gamma_{i}^{n}\right)_{\sharp}\left(\Phi^{-1}\right)_{\sharp} \tau_{K} \Phi_{\sharp}\left\langle e_{n-i}, \ldots, \widehat{e}_{n-i+j}, \ldots, e_{n}\right\rangle .
$$

For $n>0$ and $i \leq n$, let $\lambda_{i}^{n}: \Delta^{n} \longrightarrow \Delta^{n-1}$ be the definable continuous map given by $\lambda_{i}^{n}\left(\sum_{i=0}^{n} a_{i} e_{i}\right)=\sum_{j \neq i}\left(a_{j}+\frac{a_{i}}{n}\right) e_{j}$. So $\lambda_{i}^{n}$ maps $\Delta^{n}$ to $\Delta^{n-1}$ by sending the $i$-th vertex of $\Delta^{n}$ to the barycenter of $\Delta^{n-1}$. Since the image of

$$
\left(\Phi^{-1}\right)_{\sharp} \tau_{K} \Phi_{\sharp}\left\langle e_{n-i}, \ldots, \widehat{e}_{n-i+j}, \ldots, e_{n}\right\rangle
$$

is contained in the closed simplex spanned by $\left\{e_{n-i}, \ldots, \widehat{e}_{n-i+j}, \ldots, e_{n}\right\}$, after we replace in the equality above $\left(\gamma_{i}^{n}\right)_{\sharp}$ by $\left(\epsilon_{j}^{i}\right)_{\sharp}\left(\lambda_{j}^{i}\right)_{\sharp}\left(\gamma_{i}^{n}\right)_{\sharp}$ and use Lemma [5.5, we get

$$
\partial\left(\operatorname{Sd}_{i}^{K} z\right)=(\operatorname{Sd} \partial z)_{\sharp}\left(\lambda_{0}^{i}\right)_{\sharp}\left(\gamma_{i}^{n}\right)_{\sharp}\left(\Phi^{-1}\right)_{\sharp} \tau_{K} \Phi_{\sharp}\left\langle e_{n-i+1}, \ldots, e_{n}\right\rangle,
$$

which equals to $(\operatorname{Sd} \partial z)_{\sharp}\left(\gamma_{i-1}^{n}\right)_{\sharp}\left(\Phi^{-1}\right)_{\sharp} \tau_{K} \Phi_{\sharp}\left\langle e_{n-i+1}, \ldots, e_{n}\right\rangle$ since $\lambda_{0}^{i} \circ \gamma_{i}^{n}=\gamma_{i-1}^{n}$. Thus $\partial\left(\operatorname{Sd}_{i}^{K} z\right)=\operatorname{Sd}_{i-1}^{K}(\partial z)$.

To finish the proof, we will use the method of acyclic models (Theorem 2.4). Both $\mathrm{Sd}^{K}$ and $\left(1_{i}\right)_{i \leq n}$ are liftings of $1_{-1}$. On the other hand, by Lemma 5.3, if 
$\mathbf{M}=\left\{\Delta^{n}: n \in \mathbb{Z}\right\}$, then $\widetilde{S}_{*}$ is acyclic and free with a basis in M. The proof of the theorem on acyclic models (d], Chapter VI, Proposition 11.2) shows that there is a natural partial chain homotopy $T=\left(T_{i}\right)_{i \leq n}: \widetilde{S}_{*} \longrightarrow \widetilde{S}_{*+1}$ such that $\mathrm{Sd}_{i}^{K}-1_{i}=\partial T_{i}+T_{i-1} \partial$ for $i \leq n$.

The following lemma is the o-minimal analogue of Lemma 5.6.

Lemma 5.10. Suppose that $X \in \mathrm{ObjDTOP}$ and let $U$ and $V$ be open definable subsets of $X$ such that $X=U \cup V$. If $z \in \widetilde{S}_{n}(X)$, then there is a definable triangulation $(\Phi, K)$ of $\Delta^{n}$ compatible with $E^{n}$ such that $\operatorname{Sd}_{n}^{K}(z) \in \widetilde{S}_{n}(U)+\widetilde{S}_{n}(V)$.

Proof. Let $C=\left\{h^{-1}(W): W \in\{U, V\}\right.$ and $h: \Delta^{n} \longrightarrow X \in$ MorDTOP is involved in $\left.\operatorname{Sd}_{n}(z)\right\}$. See Section 2 for the notion involved. Let $(\Psi, L)$ be a definable triangulation of $\Delta^{n}$ compatible with $C$ and with $E^{n}$.

Note that, for each $h: \Delta^{n} \longrightarrow X \in$ MorDTOP involved in $\operatorname{Sd}_{n}(z)$, if $v$ is a vertex of $L, W \in\{U, V\}$ and $h \circ \Psi^{-1}(v) \in W$, then $h \circ \Psi^{-1}(|\operatorname{St} v|) \subseteq W$ where St $v$ is the star of $v$ in $L$. In fact, by the definition of $(\Psi, L)$, we have $\Psi^{-1}(t) \cap h^{-1}(W)=\emptyset$ or $\Psi^{-1}(t) \subseteq h^{-1}(W)$ for every simplex $t$ of $L$. Furthermore, since $\Psi \circ h^{-1}(W)$ is open in $|L|$, if the vertex $v$ is in $\Psi \circ h^{-1}(W)$, then $s \cap \Psi \circ h^{-1}(W) \neq \emptyset$ for each $s \in$ St $v$.

Let $(\Phi, K)$ be the definable triangulation of $\Delta^{n}$ where $K$ is the barycentric subdivision of the simplicial complex $L$ and $\Phi: \Delta^{n} \longrightarrow|L|$ is the same as $\Psi$ : $\Delta^{n} \longrightarrow|K|$ (this makes sense since $|L|=|K|$ ). Clearly, for each simplex $s$ of $K$ we have $|\bar{s}| \subseteq \mid$ St $v \mid$ for some vertex $v$ of $L$. Furthermore, for each $h: \Delta^{n} \longrightarrow X \in$ MorDTOP involved in $\operatorname{Sd}_{n}(z)$, we have $|L|=\Psi \circ h^{-1}(U) \cup \Psi \circ h^{-1}(V)$. Hence, either $h \circ \Phi^{-1}(|\bar{s}|) \subseteq U$ or $h \circ \Phi^{-1}(|\bar{s}|) \subseteq V$.

By the definition of $\operatorname{Sd}_{n}^{K}(z)$, we see that each $\sigma: \Delta^{n} \longrightarrow X \in$ MorDTOP involved in $\operatorname{Sd}_{n}^{K}(z)$ is a composition of the form $h \circ \Phi^{-1} \circ g$, where $h$ is involved in $\operatorname{Sd}_{n}(z)$ and $g: \Delta^{n} \longrightarrow|\bar{s}| \in$ MorDTOP is an affine map onto the geometric realization of the closure of an $n$-simplex $s$ of $K$. Thus $\sigma\left(\Delta^{n}\right)=h \circ \Phi^{-1}(|\bar{s}|) \subseteq W$ for some $W \in\{U, V\}$. Hence, $\operatorname{Sd}_{n}^{K}(z) \in \widetilde{S}_{n}(U)+\widetilde{S}_{n}(V)$.

Finally, after all of the above definitions and lemmas (all from [Wo]) we are ready to prove, as in $\mathrm{Wo}$, the excision axiom for the o-minimal singular homology. The argument is now similar to the corresponding proof for the excision axiom for topological singular homology: we replace the repeated barycentric subdivision operator by the subdivision operator and use Lemmas 5.9 and 5.10 instead of Lemmas 5.5 and 5.6 .

Theorem 5.11. For every $(X, A) \in \mathrm{ObjDTOPP}$ and every definable open subset $W$ of $X$ such that $\bar{W} \subseteq \AA$, the inclusion $(X-W, A-W) \longrightarrow(X, A)$ induces isomorphisms $H_{n}(X-W, A-W) \longrightarrow H_{n}(X, A)$ for all $n \in \mathbb{Z}$.

Proof. It is enough to show that if $X \in$ ObjDTOP and $U$ and $V$ are definable

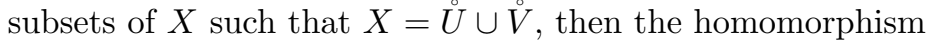

$$
\theta_{n}: H_{n}\left(\widetilde{S}_{*}(U)+\widetilde{S}_{*}(V)\right) \longrightarrow H_{n}\left(\widetilde{S}_{*}(X)\right)
$$

induced by the inclusion $\widetilde{S}_{*}(U)+\widetilde{S}_{*}(V) \longrightarrow \widetilde{S}_{*}(X)$ is an isomorphism. In fact, adapting the notation of d, page 47 , we set $\widetilde{S}_{*}\{U, V\}=\widetilde{S}_{*}(U)+\widetilde{S}_{*}(V)$ so that $H_{n}\left(\widetilde{S}_{*}\{U, V\}\right) \simeq H_{n}\left(\widetilde{S}_{*}(X)\right)$ as in d, Chapter III, 8.1 (d). Hence we also have that the homomorphism $H_{n}(U, U \cap V) \longrightarrow H_{n}(X, V)$ induced by the inclusion 
$(U, U \cap V) \longrightarrow(X, V)$ is an isomorphism. From here we get the theorem by taking $V=A$ and $U=X \backslash W$.

Below we let $\widetilde{Z}_{n}(X)=\operatorname{Ker} \partial_{n}$ and $\widetilde{B}_{n}(X)=\operatorname{Im} \partial_{n+1}$. Take $z \in \widetilde{Z}_{n}(X)$. Then by Lemma 5.10, there is a definable triangulation $(\Phi, K)$ of $\Delta^{n}$ compatible with $E^{n}$ such that $\operatorname{Sd}_{n}^{K}(z) \in \widetilde{S}_{n}(\stackrel{\circ}{U})+\widetilde{S}_{n}(\stackrel{\circ}{V}) \subseteq \widetilde{S}_{n}(U)+\widetilde{S}_{n}(V)$. By Lemma 5.9 . Sd ${ }^{K}$ is chain homotopic to the identity on $\widetilde{S}_{*}(X)$. Hence, $\operatorname{Sd}_{n}^{K}(z)+\widetilde{B}_{n}(X)=z+\widetilde{B}_{n}(X)$, and $\theta_{n}$ is surjective.

Suppose that $z_{1}+z_{2}+\widetilde{B}_{n}(U)+\widetilde{B}_{n}(V) \in \operatorname{Ker} \theta_{n}$ where $z_{1} \in \widetilde{S}_{n}(U)$ and $z_{2} \in$ $\widetilde{S}_{n}(V)$. Then there exists $w \in \widetilde{S}_{n+1}(X)$ such that $\partial w=z_{1}+z_{2}$. By Lemma 5.10, there is a definable triangulation $(\Phi, K)$ of $\Delta^{n+1}$ compatible with $E^{n+1}$ such that $\operatorname{Sd}_{n+1}^{K}(w) \in \widetilde{S}_{n+1}(U)+\widetilde{S}_{n+1}(V)$. Since $\mathrm{Sd}^{K}$ is a partial chain map, we have $\partial \operatorname{Sd}_{n+1}^{K}(w)=\operatorname{Sd}_{n}^{K}(\partial w)=\operatorname{Sd}_{n}^{K}\left(z_{1}+z_{2}\right)$. By Lemma [5.9, we have $\operatorname{Sd}_{n}^{K}\left(z_{1}+z_{2}\right)=$ $z_{1}+z_{2}+\partial T_{n}^{X}\left(z_{1}+z_{2}\right)+T_{n-1}^{X} \partial\left(z_{1}+z_{2}\right)=z_{1}+z_{2}+\partial T_{n}^{X}\left(z_{1}+z_{2}\right)$, since $\partial w=z_{1}+z_{2}$. By naturality of $T$, we have $T_{n}^{X} z_{1} \in \widetilde{S}_{n+1}(U)$ and $T_{n}^{X} z_{2} \in \widetilde{S}_{n+1}(V)$ so that $\partial T_{n}^{X} z_{1} \in$ $\widetilde{B}_{n}(U)$ and $\partial T_{n}^{X} z_{2} \in \widetilde{B}_{n}(V)$. Therefore, $z_{1}+z_{2}+\widetilde{B}_{n}(U)+\widetilde{B}_{n}(V)=\partial \operatorname{Sd}_{n+1}^{K}(w)$ is zero in $H_{n}\left(\widetilde{S}_{*}(U)+\widetilde{S}_{*}(V)\right)$ and $\theta_{n}$ is injective.

\section{O-MINIMAL COHOMOLOGY}

The results of this section are all from [e]. They are easy consequences of previous results together with classical arguments. We include the details only for completeness.

6.1. O-minimal homology with coefficients in $Q$. We will now construct the o-minimal singular homology with coefficients in an $R$-module $Q$ starting with the o-minimal singular homology with coefficients in $\mathbb{Z}$. In a similar way one can construct the o-minimal simplicial homology with coefficients in $Q$ starting from the o-minimal simplicial homology with coefficients in $\mathbb{Z}$.

Definition 6.1. Let $Q$ be an $R$-module. If $(X, A) \in \operatorname{ObjDTOPP}$, then

$$
\left(S_{*}(X, A ; Q), \partial_{*} \otimes 1_{Q}\right)
$$

where $S_{n}(X, A ; Q)=S_{n}(X, A) \otimes Q$ is a chain complex of $R$-modules. If $f$ : $(X, A) \longrightarrow(Y, B) \in$ MorDTOPP, then

$$
f_{\sharp} \otimes 1_{Q}: S_{*}(X, A ; Q) \longrightarrow S_{*}(Y, B ; Q)
$$

is a chain map. We call $\left(S_{*}(X, A ; Q), \partial_{*} \otimes 1_{Q}\right)$ the o-minimal singular chain complex with coefficients in $Q$ and we often denote $f_{\sharp} \otimes 1_{Q}$ by $f_{\sharp}$.

Theorem 6.2. The sequence of functors defined in Definition 6.1 determines an ominimal homology $\left(H_{*}, d_{*}\right)$ for DTOPP with coefficients in $Q$, called the o-minimal singular homology with coefficients in $Q$.

Proof. This is obtained from the proof of the existence of the o-minimal singular homology with coefficients in $\mathbb{Z}$ by applying the functor $-\otimes Q$. For details compare it with the classical analogue of this result presented in [d], Chapter VI, (7.1) to (7.5).

There is an analogue of Theorem 6.2 for the o-minimal simplicial homology with coefficients in $Q$. 
6.2. O-minimal cohomology. O-minimal cohomology on $C$, which is either DTOPP or DCTOPP, is defined in the following way. Recall from Section 3 that $G: C \longrightarrow C$ is the functor that sends $(X, A) \in \operatorname{Obj} C$ into $(A, \emptyset) \in \operatorname{Obj} C$ and sends $f:(X, A) \longrightarrow(Y, B) \in$ Mor $C$ into $f_{\mid A}:(A, \emptyset) \longrightarrow(B, \emptyset) \in$ Mor $C$.

Definition 6.3. An o-minimal cohomology $\left(H^{*}, d^{*}\right)$ on $C$ is a sequence $\left(H^{n}\right)_{n \in \mathbb{Z}}$ of contravariant functors from $C$ into the category of $R$-modules together with a sequence $\left(d^{n}\right)_{n \in \mathbb{Z}}$ of natural transformations $d^{n}: H^{n} \circ G \longrightarrow H^{n+1}$, such that the following axioms hold.

Homotopy Axiom. If $f, g:(X, A) \longrightarrow(Y, B) \in \operatorname{Mor} C$ and there is a definable homotopy in $C$ between $f$ and $g$, then

$$
H^{n}(f)=H^{n}(g): H^{n}(Y, B) \longrightarrow H^{n}(X, A)
$$

for all $n \in \mathbb{Z}$.

Exactness Axiom. For $(X, A) \in \operatorname{Obj} C$, if $i:(A, \emptyset) \longrightarrow(X, \emptyset)$ and $j:(X, \emptyset) \longrightarrow$ $(X, A)$ are the inclusions, then there exists a natural exact sequence

$$
\cdots \longrightarrow H^{n}(X, A) \stackrel{H^{n}(j)}{\rightarrow} H^{n}(X, \emptyset) \stackrel{H^{n}(i)}{\rightarrow} H^{n}(A, \emptyset) \stackrel{d^{n}}{\rightarrow} H^{n+1}(X, A) \longrightarrow \cdots
$$

(where the $d^{n}$ 's are induced by the natural transformations above).

Excision Axiom. For every $(X, A) \in \mathrm{Obj} C$ and every definable open subset $U$ of $X$ such that $\bar{U} \subseteq \AA$ and $(X-U, A-U) \in \operatorname{Obj} C$, the inclusion $(X-U, A-U) \longrightarrow$ $(X, A)$ induces isomorphisms

$$
H^{n}(X, A) \longrightarrow H^{n}(X-U, A-U)
$$

for all $n \in \mathbb{Z}$.

Dimension Axiom. If $X$ is a one point set, then $H^{n}(X, \emptyset)=0$ for all $n \neq 0$. The $R$-module $H^{0}(X, \emptyset)$ is called the coefficient $R$-module.

If $Q$ is the coefficient $R$-module of $\left(H^{*}, d^{*}\right)$, we will write $H^{n}(X, A ; Q)$ instead of just $H^{n}(X, A)$, and the notation $H^{n}(X, A)$ will stand for $H^{n}(X, A ; \mathbb{Z})$.

Two o-minimal cohomologies $\left(H^{*}, d^{*}\right)$ and $\left(H^{* *}, d^{\prime *}\right)$ on $C$ with a coefficient $R$ module $Q$ are isomorphic if there is a sequence $\left(\tau^{n}\right)_{n \in \mathbb{Z}}$ of natural equivalences $\tau^{n}: H^{n} \longrightarrow H^{\prime n}$ such that

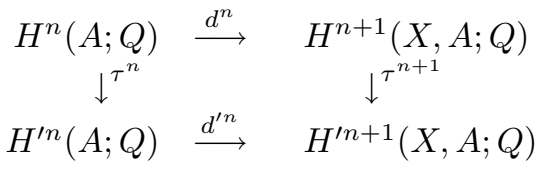

commutes for all $(X, A) \in \operatorname{Obj} C$ and for all $n \in \mathbb{Z}$.

We will now construct the o-minimal singular cohomology with coefficients in an $R$-module $Q$ starting with the o-minimal singular homology with coefficients in $\mathbb{Z}$. In a similar way one can construct the o-minimal simplicial cohomology with coefficients in $Q$ starting from the o-minimal simplicial homology with coefficients in $\mathbb{Z}$.

Definition 6.4. Let $Q$ be an $R$-module. If $(X, A) \in \operatorname{ObjDTOPP}$, then we have a cochain complex of $R$-modules

$$
\left(S^{*}(X, A ; Q), \partial_{Q}^{*}\right)
$$


where $S^{n}(X, A ; Q)=\operatorname{Hom}\left(S_{n}(X, A), Q\right)$ and $\partial_{Q}^{n}=\operatorname{Hom}\left(\partial_{n+1}, Q\right)$. If $f:(X, A) \longrightarrow$ $(Y, B) \in$ MorDTOPP, then

$$
f^{\sharp}: S^{*}(Y, B ; Q) \longrightarrow S^{*}(X, A ; Q)
$$

where $f^{\sharp}=\operatorname{Hom}\left(f_{\sharp}, Q\right)$ is a cochain map. We call $\left(S^{*}(X, A ; Q), \partial_{Q}^{*}\right)$ the o-minimal singular cochain complex with coefficients in $Q$.

Theorem 6.5. The sequence of functors defined in Definition 6.4 determines an o-minimal cohomology $\left(H^{*}, d^{*}\right)$ in DTOPP with coefficients in $Q$, called the ominimal singular cohomology with coefficients in $Q$.

Proof. This is obtained from the proof of the existence of the o-minimal singular homology with coefficients in $\mathbb{Z}$ by applying the functor $\operatorname{Hom}(-, Q)$. For details compare with the classical analogue of this result presented in [d] Chapter VI, (7.1) to $(7.5)$.

There is an analogue of Theorem 6.5 for the o-minimal simplicial cohomology with coefficients in $Q$.

\section{Comparison theOREMS}

Here we will prove the homology version of the comparison results presented in the Introduction. The cohomology version is proved dually. We will require the following lemma.

Lemma 7.1. Let $K$ be a simplicial complex, not necessarily closed, and let $L$ denote the maximal closed subcomplex of the barycentric subdivision $\mathrm{Sd} K$ of $K$. Then $\operatorname{Sd} K$ is the star of $L$ in $\mathrm{Sd} K$, and there is a definable strong deformation retraction from $|K|$ to $|L|$.

Proof. First, note that

$$
\begin{aligned}
& \operatorname{Sd} K=\left\{\left(b\left(\sigma_{0}\right), \ldots, b\left(\sigma_{n}\right)\right): \sigma_{n} \in K \text { and } \sigma_{0}<\cdots<\sigma_{n}\right\}, \\
& L=\left\{\left(b\left(\sigma_{0}\right), \ldots, b\left(\sigma_{n}\right)\right):\left\{\sigma_{0}, \ldots, \sigma_{n}\right\} \subseteq K \text { and } \sigma_{0}<\cdots<\sigma_{n}\right\},
\end{aligned}
$$

where $b(\sigma)$ denotes the barycenter of $\sigma$. Since $(b(\sigma)) \in L$ for each $\sigma \in K$, every simplex of $\operatorname{Sd} K$ is in the star of $L$.

Define $r:|K| \longrightarrow|L|$ by

$$
r(x)=\sum_{v \in \operatorname{Vert} L} \lambda_{v}(x) v / \sum_{v \in \operatorname{Vert} L} \lambda_{v}(x)
$$

where $\lambda_{v}$ is the barycentric coordinate function with respect to $v$. Then $r$ is a retraction, and, since for each $x \in|K|$ the open line segment $(x, r(x))$ lies entirely within the simplex of $K$ containing $x$, we have a strong deformation retraction from $|K|$ to $|L|$.

Proof of Theorem 1.2, Let $\left(H_{*}, d_{*}\right)$ and $\left(H^{\prime}, d_{*}^{\prime}\right)$ be two o-minimal homology theories in DTOPP with coefficients in $Q$.

Let $(X, A) \in$ ObjDTOPP. Take a definable triangulation $\left(\Phi, R_{1}\right)$ of $X$ compatible with $A$ and let $R_{2}$ be the barycentric subdivision of $R_{1}$ and $S_{2}$ the subcomplex of $R_{2}$ such that $\Phi(A)=\left|S_{2}\right|$. Let $K$ and $L$ be maximal closed subcomplexes of $R_{2}$ and $S_{2}$, respectively. Then $L \subseteq K$, and by Lemma 7.1 there are definable strong deformation retractions from $X$ to $\Phi^{-1}(|K|)$ and $A$ to $\Phi^{-1}(|L|)$. 
Let $j_{K}:|K| \longrightarrow X, j_{L}:|L| \longrightarrow A$ and $j_{K, L}:(|K|,|L|) \longrightarrow(X, A)$ be the restrictions of $\Phi^{-1}$. By the exactness axiom for o-minimal homology and arguing as in $\mathrm{dp}$, Theorem 4.3.1 and Corollary 4.3.2, we see that $H_{*}\left(j_{K}\right), H_{*}\left(j_{L}\right), H_{*}^{\prime}\left(j_{K}\right)$ and $H_{*}^{\prime}\left(j_{L}\right)$ are isomorphisms. By the exactness axiom and the five lemma, (d], Chapter I (2.9)), it follows that $H_{*}\left(j_{K, L}\right)$ and $H_{*}^{\prime}\left(j_{K, L}\right)$ are also isomorphisms.

Define $\tau_{X, A}: H_{*}(X, A ; Q) \longrightarrow H_{*}^{\prime}(X, A ; Q)$ by $\tau_{X, A}=H_{*}^{\prime}\left(j_{K, L}\right) \circ \lambda_{|K|,|L|} \circ$ $\left(H_{*}\left(j_{K, L}\right)\right)^{-1}$, where $\lambda$ is the isomorphism between $\left(H_{*}, d_{*}\right)$ and $\left(H^{\prime}, d_{*}^{\prime}\right)$ when restricted to DCTOPP. By construction and the fact that $d_{*}^{\prime} \circ \lambda_{|K|,|L|}=\lambda_{|L|} \circ d_{*}$ we have $d_{*}^{\prime} \circ \tau_{X, A}=\tau_{A} \circ d_{*}$.

We show that $\tau_{Y, B} \circ H_{*}(f)=H_{*}^{\prime}(f) \circ \tau_{X, A}$ for every $f:(X, A) \longrightarrow(Y, B)$ in MorDTOPP. Let $\left(K_{1}, L_{1}\right) \in \mathrm{ObjCSCP}$ as required for the definition of $\tau_{X, A}$. Let $\left(\Phi, R_{1}\right)$ be a definable triangulation of $Y$ compatible with $B, f\left(j_{K_{1}}\left(\left|K_{1}\right|\right)\right)$ and $f\left(j_{L_{1}}\left(\left|L_{1}\right|\right)\right)$, and let $R_{2}$ be the barycentric subdivision of $R_{1}$ and $S_{2}$ be the subcomplex of $R_{2}$ such that $\phi(B)=\left|S_{2}\right|$. Let $K$ and $L$ be maximal closed subcomplexes of $R_{2}$ and $S_{2}$, respectively. Then $L \subseteq K$, and there are definable strong deformation retractions from $Y$ to $\Phi^{-1}(|K|)$ and $B$ to $\Phi^{-1}(|L|)$, so $(K, L)$ are as required for the definition of $\tau_{Y, B}$. By [vdd], Chapter VI, Proposition 1.10, $f\left(j_{K_{1}}\left(\left|K_{1}\right|\right)\right)$ and $f\left(j_{L_{1}}\left(\left|L_{1}\right|\right)\right)$ are both closed and bounded definable sets. It follows that $f\left(j_{K_{1}}\left(\left|K_{1}\right|\right)\right)$, (resp., $f\left(j_{L_{1}}\left(\left|L_{1}\right|\right)\right)$ is a subset of $j_{K}(|K|)$ (resp., $j_{L}(|L|)$, and hence we have definable maps $f_{K_{1}}^{K}=\left(j_{K \mid f\left(j_{K_{1}}\left(\left|K_{1}\right|\right)\right)}\right)^{-1} \circ f_{\mid j_{K_{1}}\left(\left|K_{1}\right|\right)} \circ j_{K_{1}}:\left|K_{1}\right| \longrightarrow|K|$, $f_{L_{1}}^{L}=\left(j_{L \mid f\left(j_{L_{1}}\left(\left|L_{1}\right|\right)\right)}\right)^{-1} \circ f_{\mid j_{L_{1}}\left(\left|L_{1}\right|\right)} \circ j_{L_{1}}:\left|L_{1}\right| \longrightarrow|L|$ and $f_{K_{1}, L_{1}}^{K, L}:\left(\left|K_{1}\right|,\left|L_{1}\right|\right) \longrightarrow$ $(|K|,|L|)$. Note that $f \circ j_{K_{1}, L_{1}}=j_{K, L} \circ f_{K_{1}, L_{1}}^{K, L}$.

Now using the definition of $\tau_{X, A}$ and $\tau_{Y, B}$ and the fact that $\lambda_{|K|,|L|^{\circ}} H_{*}\left(f_{K_{1}, L_{1}}^{K, L}\right)=$ $H_{*}^{\prime}\left(f_{K_{1}, L_{1}}^{K, L}\right) \circ \lambda_{\left|K_{1}\right|,\left|L_{1}\right|}$, it follows that $\tau_{Y, B} \circ H_{*}(f)=H_{*}^{\prime}(f) \circ \tau_{X, A}$, as required.

Now that naturality of $\tau$ has been established, we can see that $\tau$ is independent of the choice of triangulation by considering naturality with respect to the identity $1_{(X, A)}$.

We will now give a proof of the homology version of Theorem 1.4 (the cohomology version is proved dually). This proof closely follows the proof of Theorem 1.2.

Let FTTOPP denote the category of pairs of topological spaces which are finitely triangulable over $\mathbb{R}$. The simplicial complexes in the triangulations are not required to be closed.

Before we begin the proof itself, we define a map of a simplicial complex onto itself which, under the right circumstances, retracts a neighborhood of a subcomplex onto the subcomplex. Let $K$ be a simplicial complex, $L \subseteq K, V \subseteq \operatorname{Vert} K$, and $\bar{V}=\operatorname{Vert} K-V$. Let $b(\sigma)$ denote the barycenter of $\sigma$. We define the span of $V$ in $K$, denoted $\operatorname{Sp}_{K} V$, the star of $L$ in $K$, denoted $\mathrm{St}_{K} L$, and the barycentric subdivision of $K$, denoted $\operatorname{Sd} K$, by

$$
\begin{aligned}
& \operatorname{Sp}_{K} V=\{\sigma \in K: \operatorname{Vert}(\sigma) \subseteq V\}, \\
& \operatorname{St}_{K} L=\{\sigma \in K: \exists \tau(\tau<\sigma \cap \tau \in L)\}, \\
& \operatorname{Sd} K=\left\{\left(b\left(\sigma_{0}\right), \ldots, b\left(\sigma_{n}\right)\right): \sigma_{n} \in K \text { and } \sigma_{0}<\cdots<\sigma_{n}\right\} .
\end{aligned}
$$

Note that $\operatorname{Sp}_{\operatorname{Sd} K}\{b(\sigma): \sigma \in L\}$ is the maximal closed subcomplex of $\operatorname{Sd} L$.

Define the function $\alpha_{V}:|K| \longrightarrow[0,1]$ by

$$
\alpha_{V}(x)=\sum_{v \in V} \lambda_{v}(x),
$$


where $\lambda_{v}$ is the barycentric coordinate function with respect to $v$. Note that $\alpha_{V}^{-1}(1)=\left|\operatorname{Sp}_{K} V\right|$ and $\alpha_{V}^{-1}((0,1])=\left|\operatorname{St}_{K} V\right|$. Also, $\alpha_{\bar{V}}(x)=1-\alpha_{V}(x)$.

Define the function $r_{V}:\left|\mathrm{St}_{K} V\right| \longrightarrow\left|\mathrm{Sp}_{K} V\right|$ by

$$
r_{V}(x)=\frac{1}{\alpha_{V}(x)} \sum_{v \in V} \lambda_{v}(x) v .
$$

Note that $\left(1-\alpha_{V}(x)\right) r_{\bar{V}}(x)+\alpha_{V}(x) r_{V}(x)=x$.

Let $\epsilon \in(0,1)$. Define $s_{\epsilon, V}:|K| \longrightarrow|K|$ by

$$
s_{\epsilon, V}(x)= \begin{cases}x & \text { if } \alpha_{V}(x)=0, \\ r_{V}(x) & \text { if } \alpha_{V}(x) \geq \epsilon, \\ \frac{\epsilon-\alpha_{V}(x)}{\epsilon} r_{\bar{V}}(x)+\frac{\alpha_{V}(x)}{\epsilon} r_{V}(x) & \text { if } \alpha_{V}(x) \in(0, \epsilon) .\end{cases}
$$

Then $s_{\epsilon, V}\left(\alpha_{V}^{-1}([\epsilon, 1])\right) \subseteq\left|\operatorname{Sp}_{K} V\right|$ and $s_{\epsilon, V} \simeq 1_{|K|}$. With the function $s_{\epsilon, V}$ available, we can proceed with the proof of the theorem.

Proof of Theorem 1.4. Let $\left(H_{*}, d_{*}\right)$ and $\left(H_{*}^{\prime}, d_{*}^{\prime}\right)$ be two homology theories on FTTOPP. Let $(X, A) \in$ ObjFTTOPP. By definition, there exists a triangulation $(\Phi, R)$ of $X$ compatible with $A$. As in the proof of Theorem 1.2, by taking maximal closed subcomplexes of the barycentric subdivision of $R$, we obtain closed simplicial complexes $K$ and $K^{\prime}$, with $\left|K^{\prime}\right| \subseteq|K|$, which allow us to define the map $\tau_{X, A}$, and $d_{*}^{\prime} \circ \tau_{X, A}=\tau_{A} \circ d_{*}$.

Let $f:(X, A) \longrightarrow(Y, B) \in \operatorname{MorFTTOPP}$. We wish to show that $\tau_{Y, B} \circ H_{*}(f)=$ $H_{*}^{\prime}(f) \circ \tau_{X, A}$.

There exists a triangulation $(\Psi, S)$ of $Y$ compatible with $B$. Let $S^{\prime}$ be the subcomplex such that $\left|S^{\prime}\right|=\Psi(B)$, and let $L$ and $L^{\prime}$ be maximal closed subcomplexes of the barycentric subdivisions of $S$ and $S^{\prime}$. Then $f\left(\left|K^{\prime}\right|\right) \subseteq S^{\prime} \subseteq \operatorname{St}_{\operatorname{Sd} S} L^{\prime}$. Let $V=\operatorname{Vert} L^{\prime}$. Since $f\left(\left|K^{\prime}\right|\right)$ is compact and $f\left(\left|K^{\prime}\right|\right) \subseteq \alpha_{V}^{-1}((0,1])$, there exists $\epsilon \in(0,1)$ such that $f\left(\left|K^{\prime}\right|\right) \subseteq \alpha_{V}^{-1}([\epsilon, 1])$. Then $s_{\epsilon, V} \circ f\left(\left|K^{\prime}\right|\right) \subseteq\left|L^{\prime}\right|$. Now let

$$
g=r_{\mathrm{Vert} L} \circ s_{\epsilon, V} \circ f \circ j_{K, K^{\prime}} .
$$

Then $g$ provides a map $\left(|K|,\left|K^{\prime}\right|\right) \longrightarrow\left(|L|,\left|L^{\prime}\right|\right)$ such that $j_{L, L^{\prime}} \circ g \simeq f \circ j_{K, K^{\prime}}$. Now using the definition of $\tau_{X, A}$ and $\tau_{Y, B}$ and the fact that $\lambda_{|L|,\left|L^{\prime}\right|} \circ H_{*}(g)=$ $H_{*}^{\prime}(g) \circ \lambda_{|K|,\left|K^{\prime}\right|}$, it follows that $\tau_{Y, B} \circ H_{*}(f)=H_{*}^{\prime}(f) \circ \tau_{X, A}$.

Now that the naturality of $\tau$ has been established, we can see that $\tau$ is independent of the choice of triangulation by considering naturality with respect to the identity $1_{(X, A)}$.

\section{ACKNOWLEDGEMENTS}

The first author would like to thank Gareth Owen Jones who gave an Advanced Logic Class at Oxford University based on previous versions of this paper. His comments and questions were quite useful.

\section{REFERENCES}

[bo] A.Berarducci and M.Otero O-minimal fundamental group, homology and manifolds J. London Math. Soc. 65 (2) (2002) 257-270. MR1883182 (2003b:03048)

[BCR] J.Bochnak, M. Coste and M-F. Roy Real algebraic geometry Springer-Verlag 1998. MR 1659509(2000a:14067)

[D1] H.Delfs Kohomologie affiner semialgebraischer Räume Diss. Univ. Regensburg 1980. 
[D2] H.Delfs Homology of locally semialgebraic spaces Lecture Notes in Mathematics 1484 Springer-Verlag 1991. MR1176311 (94c:14047)

[dk] H.Delfs and M.Knebusch On the homology of algebraic varieties over real closed fields J. reine angew. Math. 335 (1982) 122-163. MR667464 (84b:14010)

[dp] C.Dodson and P.Parker A user's guide to algebraic topology Kluwer Academic Publishers 1997. MR1430097(97j:55001)

[d] A.Dold Lectures on algebraic topology Springer-Verlag 1980. MR606196 (82c:55001)

[vdd] L. van den Dries Tame topology and o-minimal structures Cambridge University Press 1998. MR.1633348 (99j:03001)

[dd] J.Denef and L.van den Dries p-adic and real subanalytic sets Ann. Math. 128 (1988) 79-138. MR951508 (89k:03034)

[dm] L. van den Dries and C.Miller On the real exponential field with restricted analytic functions Israel J. Math. 85 (1994) 19-56. MR1264338 (95e:03099)

[ds1] L. van den Dries and P. Speissegger The real field with convergent generalized power series Trans. Amer. Math. Soc. 350 (11) (1998) 4377-4421. MR.1458313 (99a:03036)

[ds2] L. van den Dries and P. Speissegger The field of reals with multisummable series and the exponential function Proc. London Math. Soc. 81 (3) (2000) 513-565. MR1781147 (2002k:03057)

[e] M.Edmundo Invariance of o-minimal singular (co)homology in elementary extensions Séminaire de Structure Algébriques Ordennées n. 76 (2004) (ed., F. Delon et al.) Publications de la Université de Paris VII.

[es] S.Eilenberg and N.Steenrod Foundations of algebraic topology Princeton University Press 1952. MR0050886 (14:398b)

[k] M.Knebusch Semi-algebraic topology in the last ten years in Real Algebraic Geometry, Lecture Notes in Mathematics 1524, Springer-Verlag 1992. MR 1226239 (95d:14055)

[KPS] J.Knight, A.Pillay and C. Steinhorn Definable sets in ordered structures II Trans. Amer. Math. Soc. 295 (1986) 593-605. MR833698(88b:03050b)

[ps] Y.Peterzil and C.Steinhorn Definable compacteness and definable subgroups of o-minimal groups J. London Math. Soc. 59 (2) (1999) 769-786. MR.1709079 (2000i:03055)

[Sh] S.Shelah Classification theory and the number of non isomorphic models 2nd ed. revised, North-Holland 1990. MR.1083551 (91k:03085)

[sp] E. H. Spanier Algebraic topology McGraw-Hill 1966. MR.0210112 (35:1007)

[Wo] A.Woerheide O-minimal homology Ph.D. Thesis (1996), University of Illinois at UrbanaChampaign.

[w] A.Wilkie Model completeness results for expansions of the ordered field of real numbers by restricted Pfaffian functions and the exponential function J. Amer. Math. Soc. 9 (1996) 1051-1094. MR.1398816 (98j:03052)

CMaf, Universidade de Lisboa, Av. Prof. Gama Pinto 2, 1649-003 Lisboa, Portugal

6203 S. Evans Avenue, Chicago, Illinois 60637 